\title{
FoxO1 mediates insulin-dependent regulation of hepatic VLDL production in mice
}

\author{
Adama Kamagate, ${ }^{1}$ Shen Qu, ${ }^{1}$ German Perdomo, ${ }^{1}$ Dongming Su, ${ }^{1}$ Dae Hyun Kim, ${ }^{1}$ \\ Sandra Slusher, ${ }^{1}$ Marcia Meseck, ${ }^{2}$ and H. Henry Dong ${ }^{1}$
}

\begin{abstract}
${ }^{1}$ Rangos Research Center, Children's Hospital of Pittsburgh, Department of Pediatrics, University of Pittsburgh School of Medicine, Pittsburgh, Pennsylvania, USA. ${ }^{2}$ Department of Gene and Cell Medicine, Mount Sinai School of Medicine, New York, New York, USA.
\end{abstract}

\begin{abstract}
Excessive production of triglyceride-rich VLDL is attributable to hypertriglyceridemia. VLDL production is facilitated by microsomal triglyceride transfer protein (MTP) in a rate-limiting step that is regulated by insulin. To characterize the underlying mechanism, we studied hepatic MTP regulation by forkhead box O1 (FoxO1), a transcription factor that plays a key role in hepatic insulin signaling. In HepG2 cells, MTP expression was induced by FoxO1 and inhibited by exposure to insulin. This effect correlated with the ability of FoxO1 to bind and stimulate MTP promoter activity. Deletion or mutation of the FoxO1 target site within the MTP promoter disabled FoxO1 binding and resulted in abolition of insulin-dependent regulation of MTP expression. We generated mice that expressed a constitutively active $\mathrm{FoxO} 1$ transgene and found that increased FoxO1 activity was associated with enhanced MTP expression, augmented VLDL production, and elevated plasma triglyceride levels. In contrast, RNAi-mediated silencing of hepatic FoxO1 was associated with reduced MTP and VLDL production in adult mice. Furthermore, we found that hepatic FoxO1 abundance and MTP production were increased in mice with abnormal triglyceride metabolism. These data suggest that FoxO1 mediates insulin regulation of MTP production and that augmented MTP levels may be a causative factor for VLDL overproduction and hypertriglyceridemia in diabetes.
\end{abstract}

\section{Introduction}

Hypertriglyceridemia is a prominent pathological feature of metabolic syndrome and is characterized by augmented production and/or retarded clearance of triglyceride-rich (TG-rich) particles, such as VLDL and chylomicrons $(1,2)$. Subjects with hypertriglyceridemia manifest elevated plasma TG and reduced HDL cholesterol levels, accompanied by high levels of small dense LDL particles, an atherogenic profile that constitutes a major risk factor for artery blockage and cardiovascular disease $(2,3)$. While the pathophysiology of hypertriglyceridemia is poorly understood, its close association with visceral adiposity and type 2 diabetes implicates insulin resistance as a causative factor for hypertriglyceridemia (3-5). VLDL is assembled and produced in the liver, which depends on substrate availability and is sensitive to insulin inhibition $(6,7)$. In visceral obesity and type 2 diabetes, aberrant insulin action in coalition with increased influx of FFAs into liver promotes excessive VLDL-TG production, contributing to the pathogenesis of hypertriglyceridemia $(3,4,8)$. Nonetheless, the molecular basis that links insulin resistance to hepatic VLDL-TG overproduction remains obscure.

Crucial for hepatic VLDL production is microsomal TG transfer protein (MTP), an ER resident protein that facilitates the transfer of lipids to nascent apoB in liver (9-11). There are clinical data indicating that a lack of MTP activity, caused by genetic lesions in the MTTP gene, results in abetalipoproteinemia, a rare autosomal recessive disease that is characterized by defects in the assembly and secretion of TG-rich particles, abnormalities in lipid metabolism,

Nonstandard abbreviations used: ACC, acetyl CoA carboxylase; FAS, fatty acid synthase; FoxO1, forkhead box O1; G6Pase, glucose-6-phosphatase; HNF4 $\alpha$, hepatocyte nuclear factor $4 \alpha$; IRE, insulin response element; MTP, microsomal TG transfer protein; NEFA, nonesterified fatty acid; PEPCK, phosphoenolpyruvate carboxykinase; SREBP-1c, sterol regulatory element binding protein-1c; TG, triglyceride.

Conflict of interest: The authors have declared that no conflict of interest exists. Citation for this article: J. Clin. Invest. 118:2347-2364 (2008). doi:10.1172/JCI32914 and severe vitamin deficiency (12-16). Preclinical studies indicate that hepatic MTP overproduction, as a result of transgenic MTP expression or adenovirus-mediated MTP production in liver, results in excessive VLDL-TG secretion and elevated plasma TG levels in mice (17). MTP haploinsufficiency is associated with reduced plasma TG levels, due to diminished hepatic VLDL secretion in MTP heterozygous mice (15). Pharmacological inhibition of MTP activity is shown to reduce VLDL production and decrease plasma cholesterol levels in subjects with familial hypercholesterolemia (18). There is emerging evidence that diminished MTP activity is a compounding factor for advanced alcoholic liver disease (19).

Despite the critical role of MTP in hepatic VLDL production and plasma TG metabolism, the molecular events that control MTP expression in response to insulin action and other metabolic effectors remain poorly characterized. Studies in insulin-resistant non-diabetic obese Zucker rats and high-fructose diet-induced hyperlipidemic hamsters indicate that hepatic MTTP mRNA levels are significantly elevated, coinciding with increased TG secretion $(4,20,21)$. These data are consistent with the findings that MTP is negatively regulated by insulin (22-24). Recent studies in HepG2 cells show that insulin-dependent inhibition of MTP and VLDL apoB production involves the activation of MAPK $(22,25)$. However, targeted blockage of MAPK activity only results in partial inhibition of insulin-mediated reduction in VLDL apoB production $(22,25)$, suggesting that other mechanisms are involved in insulin-dependent inhibition of MTP and VLDL apoB production in liver. Indeed, Siri et al. (26) show that hepatic VLDL and apoB production was markedly elevated without significantly altering hepatic MTTP mRNA levels in hyperlipidemic mice that overexpress human apoB but lack brown adipose tissue. A more recent study by Ota et al. shows that hepatic VLDL-TG and apoB secretion is inhibited in response to ER stress, contributing to hepatic steatosis in mice with high-fat diet-induced obesity (27). 
A
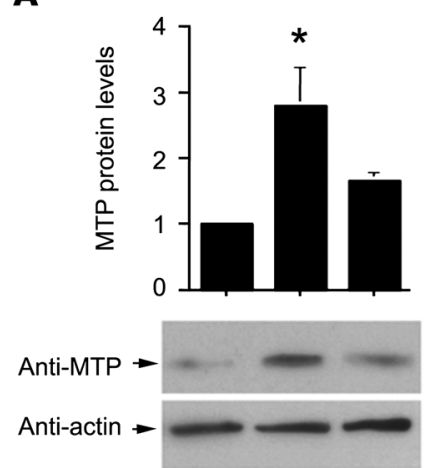

Vector Control FoxO1 FoxO1

Insulin $\quad-\quad-\quad+$
B
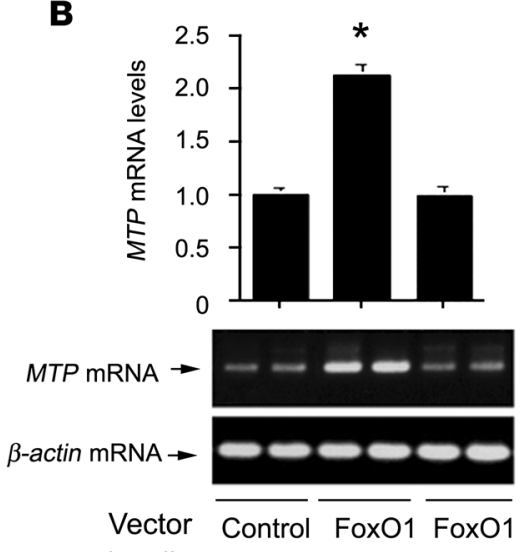

Insulin - - +

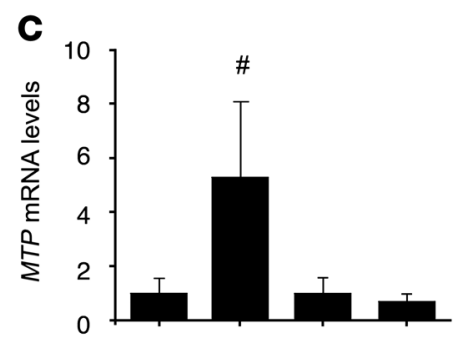

Control + $\mathrm{FoxO1}-\quad+\quad+\quad+$ Akt-CA - $\quad-\quad+\quad-$ Insulin $-\quad-\quad-\quad+$

$\mathbf{F}$



G
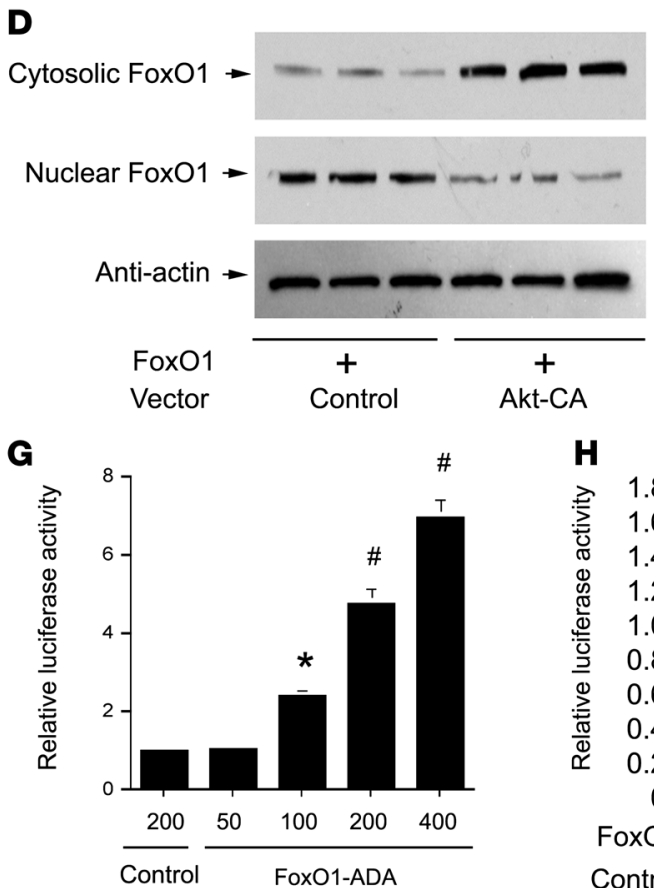

J

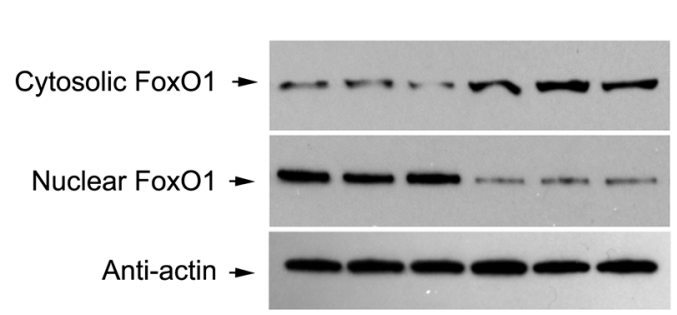

Fox01 vector ++++++

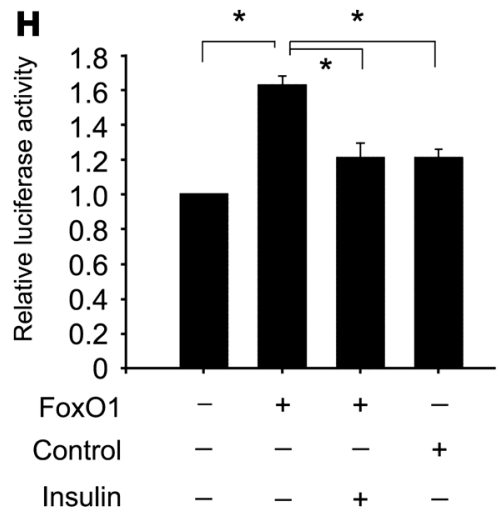

Insulin $--\quad+++$
K
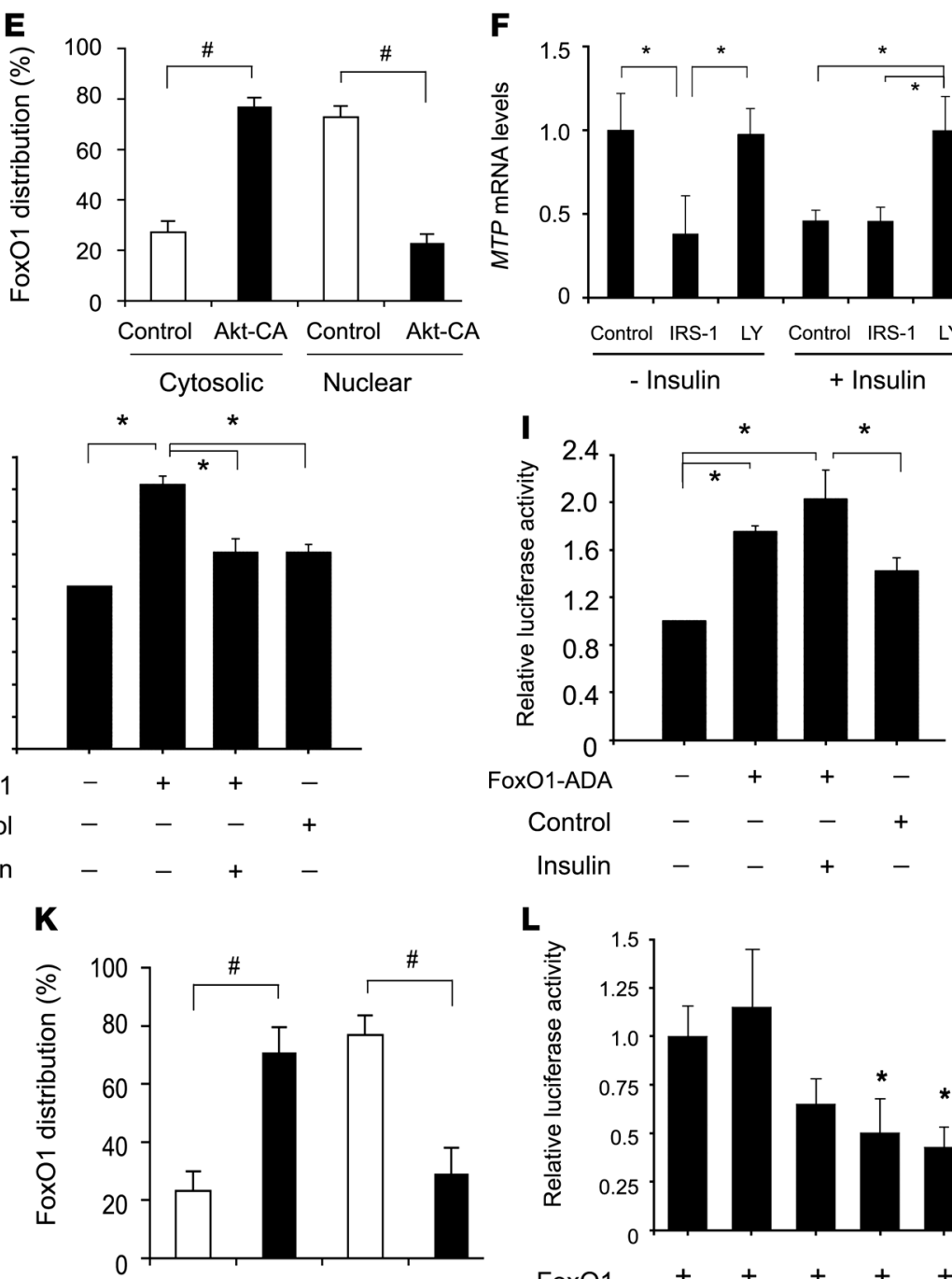

L

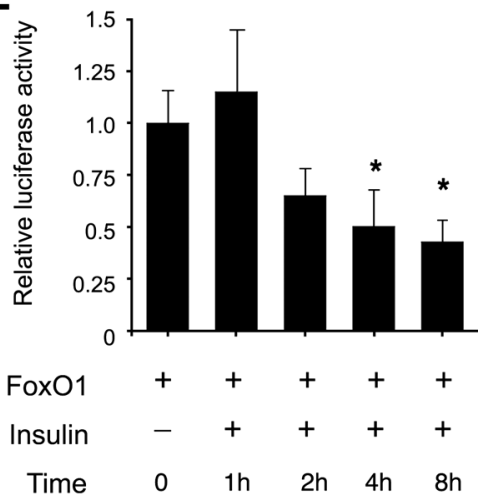




\section{Figure 1}

Effect of FoxO1 on MTP expression in HepG2 cells. (A) Hepatic MTP protein levels. (B) Hepatic MTTP mRNA levels. (C) Hepatic MTTP mRNA levels. HepG2 cells were transduced with control LacZ or FoxO1 vector or FoxO1 plus CA-Akt vector (MOI, 25 pfu/cell), followed by the determination of MTTP mRNA levels after 24-h incubation in the presence or absence of insulin (100 nM). (D) FoxO1 subcellular distribution. FoxO1 vector-transduced HepG2 cells were incubated with control or CA-Akt vector for $24 \mathrm{~h}$, followed by immunoblot analysis of FoxO1 protein levels in cytosolic and nuclear fractions (E). (F) Hepatic MTTP mRNA levels. HepG2 cells were treated with PI3K activator IRS-1 (Y608) peptide (IRS-1, $1 \mu \mathrm{M})$ or inhibitor LY294002 (LY, $10 \mu \mathrm{M})$ in the presence and absence of insulin for $24 \mathrm{~h}$. (G) FoxO1 induction of MTP promoter activity. HepG2 cells were cotransfected with $2 \mu \mathrm{g}$ each $\mathrm{pGH} 11$ and $\mathrm{pCMV}$-LacZ vectors in the presence of Adv-FoxO1-ADA vector at doses ranging from 50 to $400 \mathrm{pfu} / \mathrm{cell}$, followed by luciferase activity assay after 24-h incubation. Likewise, HepG2 cells were cotransfected with pGH11 and pCMV-LacZ plasmids together with FoxO1 $(\mathbf{H})$ or FoxO1-ADA (I) vector at a fixed dose (MOI, 100 pfu/cell) in the presence and absence of insulin for the determination of luciferase activity. (J) FoxO1 subcellular distribution. FoxO1 vector-treated HepG2 cells were incubated in the absence or presence of insulin for $30 \mathrm{~min}$, followed by immunoblot analysis of FoxO1 in cytosolic and nuclear fractions (K). (L) Insulin inhibition of MTP promoter activity. FoxO1 vector-transduced HepG2 cells were transfected with MTP promoter-directed reporter system in the presence or absence of insulin. Cells were assayed for luciferase activity at different times. Data represent $3-5$ experiments. ${ }^{*} P<0.05$, ${ }^{\#} P<0.001$ versus controls.

To better understand the molecular basis of insulin-dependent regulation of hepatic MTP and VLDL production, we studied hepatic regulation of MTP by forkhead box O1 (FoxO1), a transcription factor that mediates the inhibitory action of insulin on target gene expression (28-30). FoxO1 stimulated hepatic MTP expression, and this effect was counteracted by insulin. FoxO1 gain-of-function is associated with enhanced MTP expression, correlating with augmented hepatic VLDL production and elevated plasma TG levels in FoxO1 transgenic mice. FoxO1 loss-of-function, caused by RNAi-mediated depletion of Foxo1 mRNA in liver resulted in reduced hepatic MTP and VLDL production in diabetic $d b / d b$ and FoxO1 transgenic mice. Our studies suggest that insulin signaling through FoxO1 plays an important role in regulating hepatic MTP expression and VLDL production.

\section{Results}

Effect of FoxO1 on hepatic MTP expression. Insulin inhibits hepatic MTP expression $(23,24)$, but the underlying mechanism and physiology remains undefined. To dissect the mechanism of insulin-dependent inhibition of hepatic MTP expression, we delivered FoxO1 cDNA into HepG2 cells, followed by determination of intracellular MTP mRNA and protein levels in the presence and absence of insulin in culture medium. Adenovirus-mediated FoxO1 production resulted in about 3-fold induction of MTP protein (Figure 1A) and 2-fold induction of MTTP mRNA levels (Figure 1B) in HepG2 cells. This effect was reversed by insulin, as MTP mRNA and protein levels were suppressed to basal levels after the addition of insulin into culture medium.

To address the hypothesis that insulin signaling through FoxO1 plays an important role in regulating hepatic MTP expression, we studied FoxO1-mediated induction of hepatic MTTP mRNA expression in the absence and presence of CA-Akt, a constitutively active Akt that has been shown to phosphorylate its targets independently of insulin (31). As shown in Figure 1C, transduction of HepG2 cells with FoxO1 vector resulted in a marked induction of hepatic MTTP mRNA. However, this effect was completely abolished in HepG2 cells that were cotransduced with both FoxO1 and CA-Akt vectors independently of insulin.

To determine whether CA-Akt-mediated inhibition of FoxO1 activity is through Akt-dependent nuclear exclusion, we prepared cytoplasmic and nuclear fractions of HepG2 cells that were pretransduced with FoxO1 vector alone or in combination with CAAkt vector, followed by semi-quantitative immunoblot assay using an anti-FoxO1 antibody. As shown in Figure 1D, FoxO1 proteins were detected predominantly in nuclear fractions in the absence of
CA-Akt. In the presence of CA-Akt production in cells, FoxO1 was located in cytoplasmic fractions. These observations were confirmed by the quantification of FoxO1 subcellular distribution. In response to CA-Akt production, FoxO1 underwent a quantitative shift from the nucleus to cytoplasm (Figure 1E). This effect correlated with the suppression of MTTP mRNA expression in HepG2 cells that were cotransduced with FoxO1 and CA-Akt vectors (Figure 1C).

To examine the effect of PI3K on hepatic MTTP expression, we used PI3K-specific activator IRS-1 (Y608) peptide and inhibitor LY294002 to treat HepG2 cells, followed by the determination of hepatic MTTP mRNA levels in response to insulin action. IRS-1 (Y608) peptide was shown to suppress hepatic MTTP expression (Figure 1F). This effect occurred irrespective of insulin addition into culture medium and correlated with the ability of IRS-1 (Y608) peptide to stimulate PI3K activity. In the presence of PI3K inhibitor LY294002, the ability of insulin to regulate MTTP expression was abolished, as evidenced by the lack of alterations in hepatic MTTP mRNA levels in HepG2 cells in the presence and absence of insulin (Figure 1F). As control, insulin addition resulted in a marked reduction in hepatic MTP expression in the absence of LY294002 (Figure 1F). These results demonstrate that activation of PI3K decreased MTTP mRNA expression, which is reminiscent of the inhibitory effect of insulin on hepatic MTTP expression. In contrast, blocking of PI3K activity resulted in abolition of insulin action on hepatic MTTP expression.

To determine whether the MTTP gene is a FoxO1 target, we cloned the mouse MTP promoter into a luciferase reporter system and assayed the MTP promoter activity in response to FoxO1 production. As shown in Figure 1G, adenovirus-mediated FoxO1-ADA production stimulated MTP promoter activity in a dose-dependent manner in HepG2 cells. To study whether FoxO1-mediated induction of MTP promoter activity is subject to insulin inhibition, we transduced HepG2 cells at a fixed dose of FoxO1 vector with and without insulin in culture medium. As shown in Figure $1 \mathrm{H}$, adenovirus-mediated FoxO1 production markedly elevated MTP promoter activity. Addition of insulin into culture medium resulted in abolition of FoxO1-mediated stimulation of MTP promoter activity in HepG2 cells. To corroborate these findings, we transduced FoxO1-ADA vector into HepG2 cells. Due to mutations at 3 conserved phosphorylation sites (T24, S253, and S316) in FoxO1 polypeptide, FoxO1-ADA is unable to undergo insulindependent phosphorylation and nuclear exclusion $(31,32)$. As a result, FoxO1-ADA is permanently located in the nucleus, resulting in constitutive target gene expression. As shown in Figure 1I, MTP promoter activity, defined as the relative level of luciferase activity, 
A

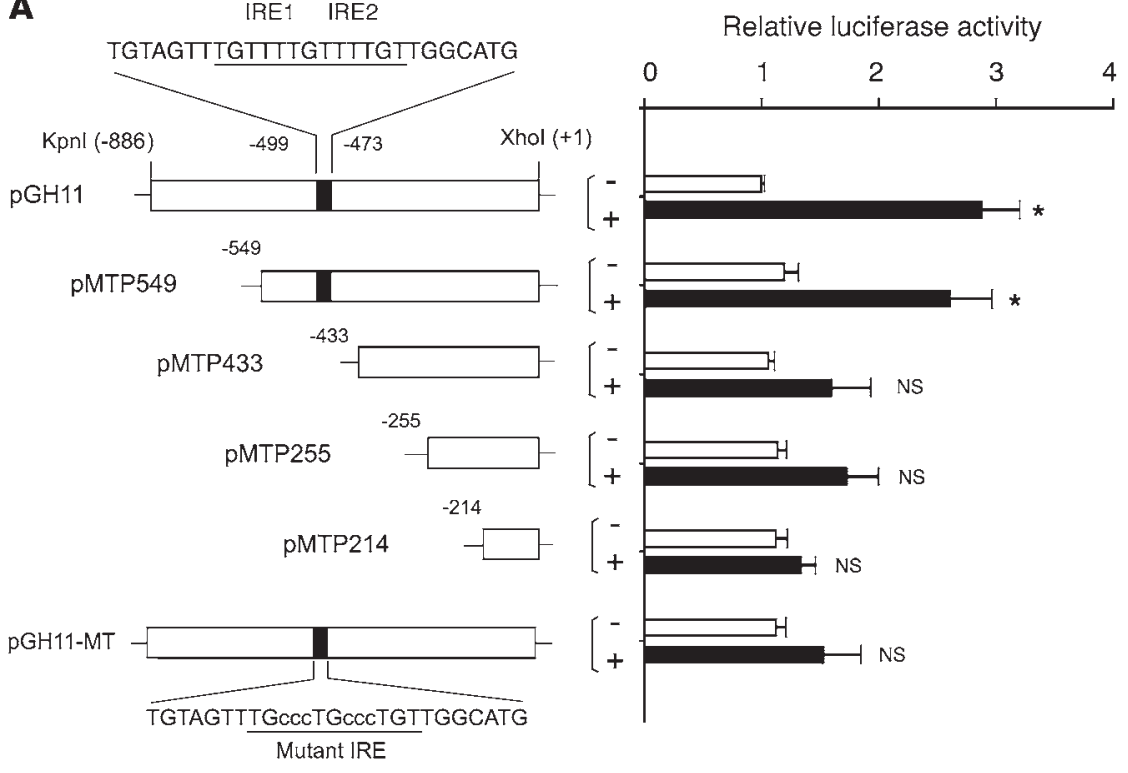

Figure 2

Effect of FoxO1 on MTP promoter activity. (A) HepG2 cells in 6-well microplates were cotransfected with pCVM5-LacZ vector plus wild-type or mutant MTP promoter-directed luciferase reporter systems in the presence of Adv-null vector (-) or Adv-FoxO1 vector (+) at a fixed dose (MOI, 200 pfu/cell). After 24-h incubation, the luciferase activities were normalized to $\beta$-gal activity and then determined and compared between control and FoxO1 conditions. Data were obtained from 8 experiments. (B) Response of wild-type and mutant MTP promoters to insulin. HepG2 cells were transfected with test plasmids using pCMV-LacZ as a control and transduced with either Adv-null or AdvFoxO1 vector in the presence and absence of insulin $(100 \mathrm{nM})$. The relative luciferase activity for each construct was determined after 24-h incubation. Data were obtained from 4-9 experiments. ${ }^{*} P<0.001$ versus control.

\section{B}

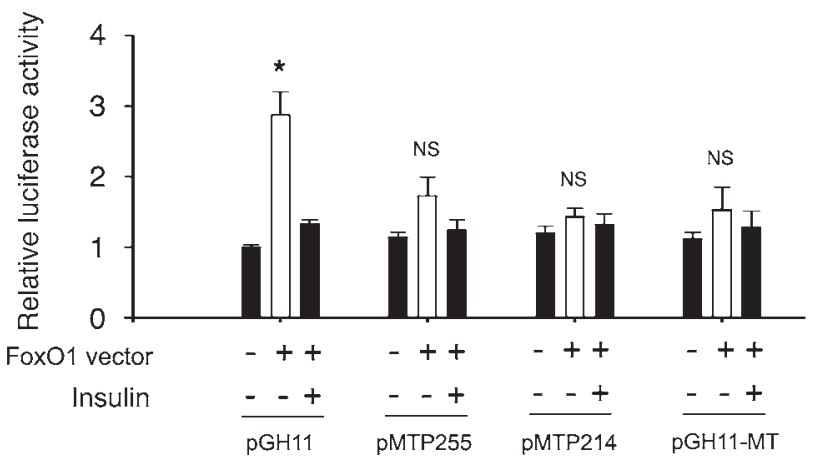

was significantly enhanced by adenovirus-mediated FoxO1-ADA production in HepG2 cells. Unlike FoxO1-mediated induction of MTP promoter activity that was counteracted by insulin, FoxO1ADA-mediated induction of MTP promoter activity remained unchanged despite insulin addition into the culture medium.

To consolidate these findings, we determined the acute effect of insulin on FoxO1 trafficking. HepG2 cells pretransduced with FoxO1 vector were incubated in the absence or presence of insulin for $30 \mathrm{~min}$, followed by quantitative analysis of FoxO1 subcellular distribution in the cytoplasm compared with distribution in the nucleus. In keeping with previous observations $(31,32)$, about $80 \%$ of total cellular FoxO1 proteins were translocated from the nucleus to the cytoplasm within $30 \mathrm{~min}$ following insulin addition into culture medium (Figure 1, J and K).

To determine time-dependent inhibition of MTP expression by insulin, we assayed MTP promoter activity using luciferase as a reporter. HepG2 cells were transfected with the MTP promoter-directed luciferase expression system in the presence of FoxO1 expression. After 24 -h incubation, insulin $(100 \mathrm{nM})$ was added into cultured medium and aliquots of cells were harvested at different times for the determination of luciferase activity at different times. Insulin addition resulted in a time-dependent inhibition of MTP promoter activity in HepG2 cells, with the establishment of half-maximal inhibition within $2-4 \mathrm{~h}$ (Figure 1L). These data provide proof-ofconcept that MTP promoter activity is subject to insulin inhibition in a time-dependent manner. The observed delay in insulin-mediated inhibition of MTP promoter activity was likely due to the relative stability of luciferase proteins in cultured HepG2 cells (22).

Characterization of FoxO1 target site within MTP promoter. To define FoxO1 target site in the MTP promoter, we generated a series of promoter variants with different deletions of the upstream region of the MTP promoter, followed by subcloning into the luciferase reporter system. Following transfection into HepG2 cells, the activity of promoter variants was determined in the presence and absence of FoxO1 production. As shown in Figure 2A, deletion up to $-549 \mathrm{nt}$ in the MTP promoter (pMTP549) did not alter the promoter activity in response to FoxO1, as similar levels of luciferase activity were detected in HepG2 cells that were pretransduced with pMTP549 and its wild-type counterpart in the presence of FoxO1 production. Further deletion up to $-214 \mathrm{nt}$ in the MTP promoter rendered the promoter variant (PMTP214) unresponsive to FoxO1-mediated induction. These results confined the putative FoxO1 target site to a small region (-549/-214 nt) in the MTP promoter. To further characterize FoxO1 target site, we determined the activity of 2 additional promoter variants with DNA deletions up to $-433 \mathrm{nt}$ (pMTP433) and -255 nt (pMTP255), respectively, using the luciferase reporter assay. As shown in Figure 2A, these 2 promoter variants remained indifferent in response to $\mathrm{FoxO} 1$ production in HepG2 cells. As a result, the FoxO1 target site was localized to the -549/-433-nt region of the MTP promoter. 
A

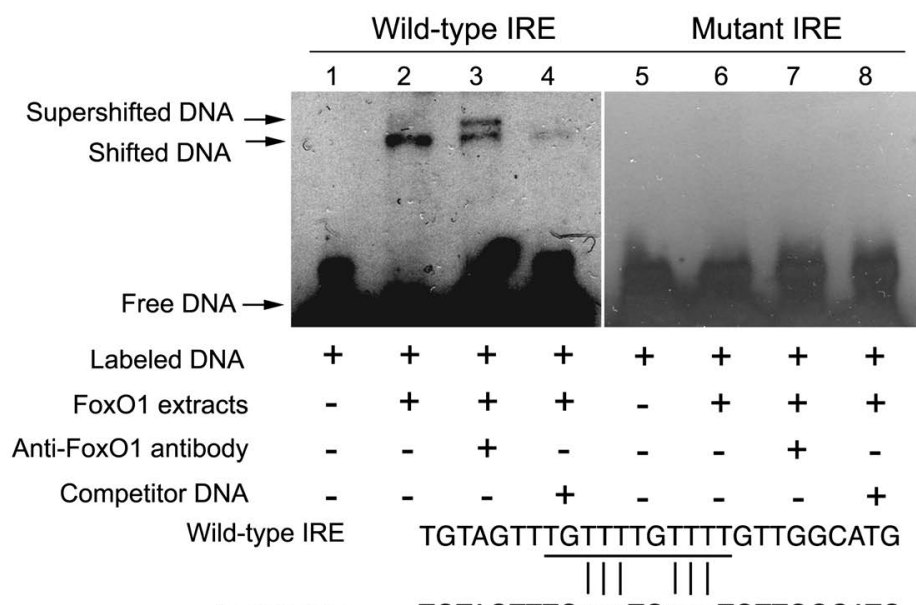

B

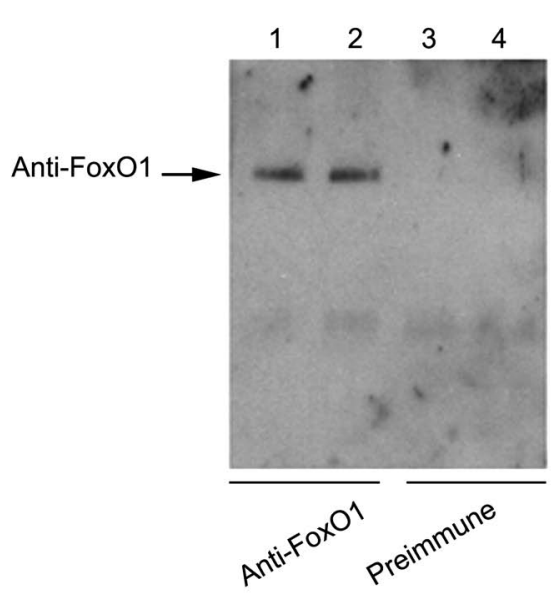

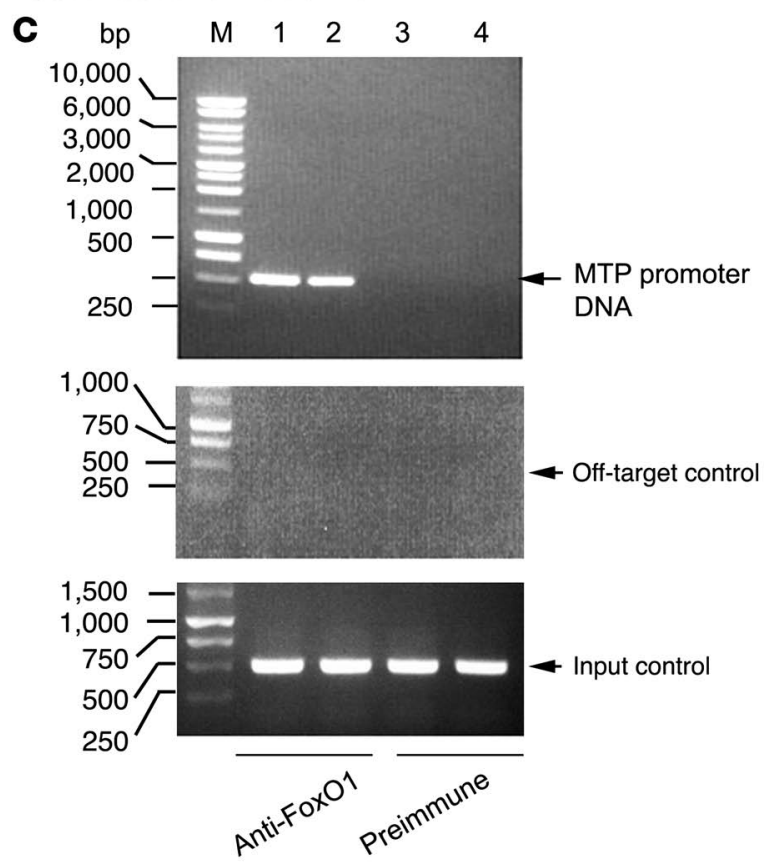

Figure 3

Molecular interaction between FoxO1 and MTP promoter. (A) EMSA for assaying FoxO1 binding to DNA. HepG2 cells were transduced with FoxO1 vector at an $\mathrm{MOI}$ of $200 \mathrm{pfu} / \mathrm{cell}$. Cells were lysed for the preparation of nuclear protein extracts $24 \mathrm{~h}$ after transduction. Aliquots of FoxO1 protein extracts $(5 \mu \mathrm{g})$ were incubated with a biotin-labeled DNA probe, followed by chemiluminescent EMSA. DNA probe was derived from a 27-bp DNA covering the consensus IRE (-499/-473 nt) of the mouse MTP promoter, shown in lanes 1-4. A mutant DNA with an altered IRE motif was used as a control, shown in lanes 5-8. In addition, ChIP assay was used to assay for FoxO1 binding to DNA in cells. HepG2 cells were transfected with pGH11 in the presence of FoxO1 vector at an MOI of 100 pfu/cell in triplicate. After 24-h incubation, cells were cross-linked with $1 \%$ formaldehyde, followed by ChIP assay using rabbit anti-FoxO1 antibody (lanes 1 and 2) or preimmune IgG as a control (lanes 3 and 4). Immunoprecipitates were subjected to immunoblot assay using anti-FoxO1 antibody (B), and to PCR analysis using a pair of primers flanking the IRE sequence in the MTP promoter (C). As a positive control, aliquots of input DNA samples (1 $\mu$ l) were used in PCR analysis. As a negative control, the immunoprecipitates were subjected to PCR analysis using a pair of off-target primers flanking a distal region (-3,528/-3,045 nt) devoid of the consensus IRE motif at $3 \mathrm{~kb}$ upstream of the MTP promoter.

These data spurred us to perform sequence analysis of the MTP promoter $(2 \mathrm{~kb})$ for the FoxO1 DNA binding motif (T[G/ A]TTT[T/G]), known as the "insulin response element" (IRE), which is commonly present in the promoter of genes whose expressions are inhibited by insulin $(29,31,33,34)$. We detected 2 IRE motifs that were intertwined within a DNA region (-549/-433 nt) of the MTP promoter (Figure 2A). Sequence analysis also revealed the presence of the IRE motifs within the human $(-542 /-535 \mathrm{nt})$ and rat (-332/-325 nt) MTP promoters, suggesting a conserved mechanism for insulin-dependent regulation of hepatic MTP expression among species. To determine whether this IRE motif was responsible for insulin-dependent regulation of MTP expression, we assayed the activity of MTP promoter variants in response to FoxO1 production in HepG2 cells in the presence and absence of insulin. As shown in Figure 2B, adenovirus-mediated production of FoxO1 resulted in about a 3 -fold induction in the activ- 


\section{A}

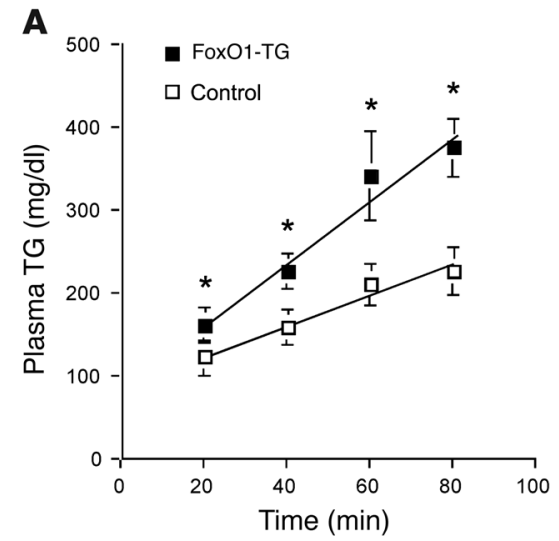

C

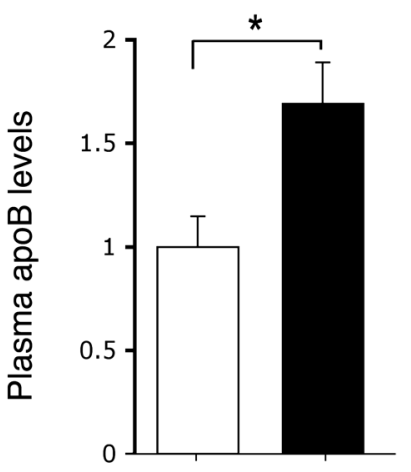

Anti-apoB -

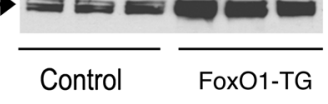

E

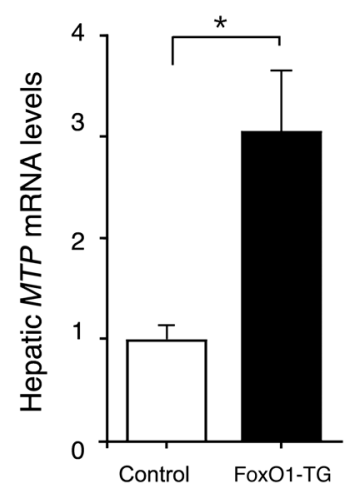

G

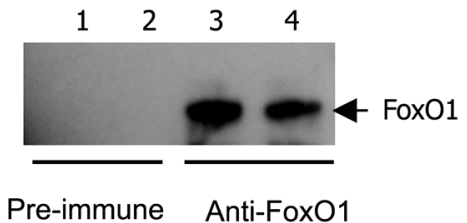

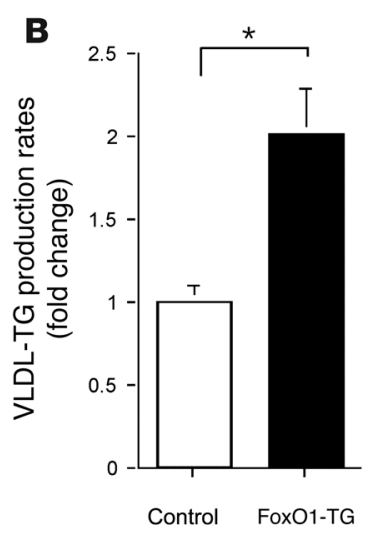

D

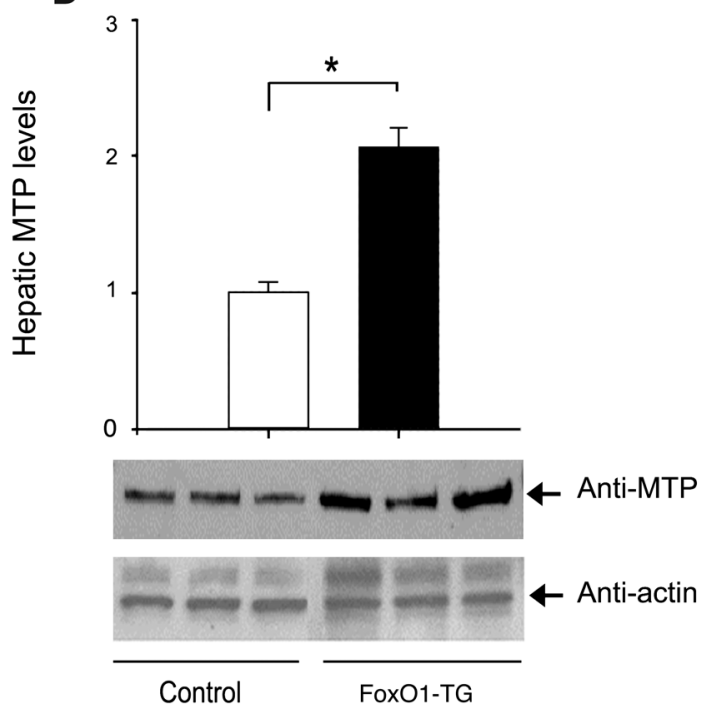

$\begin{array}{lllllll}\mathbf{F} & \text { bp } & M & 1 & 2 & 3 & 4\end{array}$

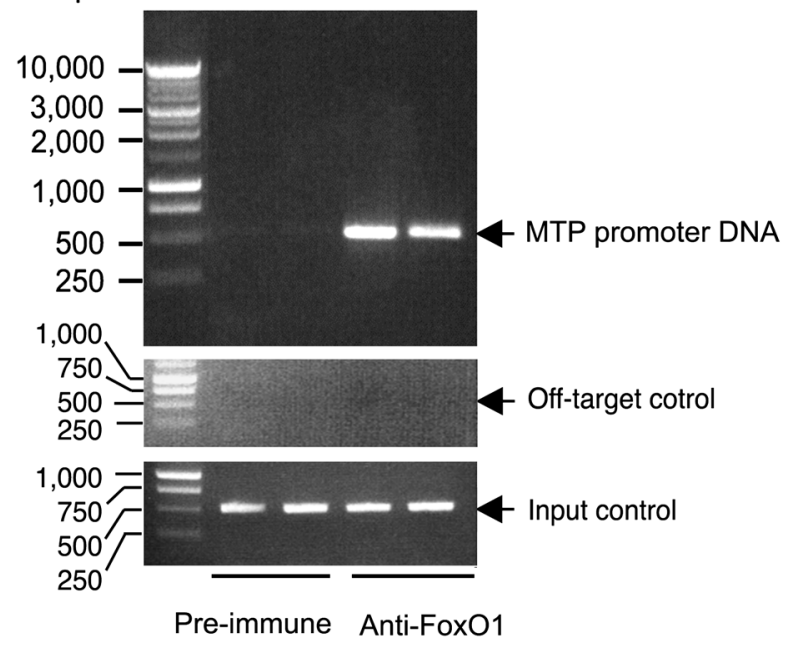




\section{Figure 4}

VLDL production in FoxO1 ${ }^{\mathrm{S} 253 \mathrm{~A}}$ transgenic versus control mice. Male FoxO1 ${ }^{\mathrm{S} 253 \mathrm{~A}}$ transgenic mice $(n=6)$ and control littermates $(n=6)$ at 6 months of age were fasted for $5 \mathrm{~h}$, followed by intravenous injection of tyloxapol at $500 \mathrm{mg} / \mathrm{kg}$ body weight per mouse to inhibit plasma VLDL clearance. (A) Aliquots of tail vein blood were taken at different times for the determination of plasma TG levels. (B) The relative rates of VLDL secretion are defined by the slopes of linear increases of plasma TG as a function of time following intravenous injection of tyloxapol. (C) Aliquots of plasma (20 $\mu \mathrm{g}$ protein) obtained from mice at $80 \mathrm{~min}$ after tyloxapol injection were analyzed by semiquantitative immunoblot assay using anti-apoB antibody for the determination of plasma apoB secretion. Mice were sacrificed at 6 months of age, and liver tissues were subjected to semiquantitative immunoblot analysis for the determination of hepatic MTP protein abundance (D), and separately to real-time quantitative RT-PCR for the determination of hepatic MTTP mRNA levels (E). ${ }^{*} P<0.05$ versus control. In addition, liver tissues were subjected to ChIP analysis using preimmune rabbit serum (lanes 1 and 2) and rabbit anti-FoxO1 antibody (lanes 3 and 4). The resulting immunoprecipitates were analyzed by PCR (F) and immunoblot (G) assays as described in Figure 3.

ity of wild-type MTP promoter. This induction was inhibited by insulin. After the IRE motif was deleted, the resulting mutant promoters (pMTP255 and PMTP214) were no longer responsive to FoxO1-mediated induction and insulin-mediated inhibition of promoter activities (Figure $2 \mathrm{~B}$ ). To underpin these findings, we altered the core IRE motif by site-directed mutagenesis. The resulting mutant MTP promoter was assayed for its ability to mediate FoxO1 induction in the presence and absence of insulin. As shown in Figure 2, mutations in the IRE abolished FoxO1mediated induction and simultaneously abrogated insulin-mediated inhibition of MTP promoter activity.

FoxO1 association with MTP promoter. To test the ability of this IRE motif to bind FoxO1, we conducted EMSA to determine the molecular interaction between IRE DNA and FoxO1 protein. Nuclear protein extracts, prepared from FoxO1-expressing HepG2 cells, were mixed with a 27-bp IRE DNA sequence that was prelabeled with biotin, followed by chemiluminescent EMSA. As shown in Figure 3A, FoxO1 addition to the reaction mixture caused a significant retardation of the IRE DNA in a $6 \%$ native polyacrylamide gel. To assay the specificity of FoxO1 binding to IRE DNA, anti-FoxO1 antibody or, alternatively, nonlabeled IRE DNA at 100-fold higher concentrations were added to the reaction mixture as competitors. Inclusion of anti-FoxO1 antibody resulted in a supershifted DNA band in the gel. In contrast, inclusion of excess nonlabeled competitive DNA diminished the shifted DNA band. As control, we performed the same EMSA using a mutant version of the IRE DNA containing 6 base substitutions within the IRE motif. Alterations of the consensus IRE motif abrogated its ability to bind FoxO1, as the mutant IRE DNA was negative when tested in the presence of FoxO1 by EMSA.

To corroborate the above findings, we performed ChIP assay to determine the association between FoxO1 and MTP promoter DNA in HepG2 cells. This assay allows the detection of potential interaction of proteins with target chromatin DNA in living cells. Due to extremely low levels of FoxO1 expression in HepG2 cells, we transduced HepG2 cells with wild-type FoxO1 vector, followed by ChIP assay using rabbit anti-FoxO1 antibody or preimmune rabbit sera. The immunoprecipitates were subjected to immunoblot assay for the detection of immunoprecipitated FoxO1 and PCR analysis for the visualization of coimmunoprecipitated promoter DNA. As shown in Figure 3B, specific bands corresponding to FoxO1 protein were detected in the complexes that were immunoprecipitated by anti-FoxO1. Using primers flanking the IRE motif within the MTP promoter, we detected by PCR a sequence-specific DNA corresponding to the proximal region $(-549 /+1 \mathrm{nt})$ of the MTP promoter in the same immunoprecipitates by anti-FoxO1 (Figure 3C). In contrast, the immunoprecipitates derived from pre-immune sera were negative when analyzed by immunoblot and PCR assays. As an input control, aliquots of cell lysates $(1 \mu \mathrm{l})$ prior to immunoprecipitation were subjected to the same PCR analysis. Specific DNA bands corresponding to the MTP promoter were detected (Figure 3C). In addition, we performed PCR analysis using a pair of off-target primers flanking a distal region $(-3,528 /-3,045 \mathrm{nt})$ that is devoid of the consensus IRE motif at 3-kb upstream of the MTP promoter. No specific DNA was amplified in the immunoprecipitates by pre-immune IgG or anti-FoxO1 antibody.

Effect of FoxO1 on hepatic VLDL production. To better understand the underlying physiology of FoxO1-mediated regulation of MTP expression, we studied the effect of FoxO1 on hepatic VLDL production. MTP catalyzes the transport of TG, cholesteryl ester, and phospholipids to nascent polypeptides of apoB, a rate-limiting step for hepatic VLDL assembly and secretion $(9,12)$. Our hypothesis was that a gain-of-function of FoxO1 would enhance MTP production and augment hepatic VLDL secretion. To test this hypothesis, we determined hepatic VLDL-TG secretion rates in FoxO $1^{\mathrm{S} 253 \mathrm{~A}}$ transgenic mice expressing a constitutively active FoxO1 allele $(35,36)$. Male FoxO $1^{\mathrm{S} 253 \mathrm{~A}}$ transgenic mice $(n=6$; 6 months of age) and sex- and age-matched control littermates $(n=6)$ were fasted for $5 \mathrm{~h}$, followed by intravenous injection of tyloxapol (500 mg/kg per mouse) to inhibit plasma VLDL clearance. Aliquots of tail vein blood $(25 \mu \mathrm{l})$ were sampled at different times for the determination of plasma TG levels. As shown in Figure 4, A and B, FoxO1 ${ }^{\mathrm{S} 253 \mathrm{~A}}$ transgenic mice exhibited significantly higher rates of hepatic VLDL-TG production when compared with control littermates. To buttress these findings, we subjected aliquots of sera ( $20 \mu \mathrm{g}$ of serum protein) at $80 \mathrm{~min}$ after tyloxapol administration to immunoblot assays using anti-apoB antibody. As shown in Figure 4C, significantly higher apoB100 levels were detected in the sera of FoxO $1^{\mathrm{S} 253 \mathrm{~A}}$ transgenic mice. To correlate hepatic VLDL-TG production with hepatic MTP expression, we determined hepatic MTP protein levels by semiquantitative immunoblot using rabbit anti-MTP antibody. As shown in Figure $4 \mathrm{D}$, significantly higher MTP protein levels were detected in livers of FoxO1 ${ }^{\mathrm{S} 253 \mathrm{~A}}$ transgenic mice. In addition, we determined hepatic MTTP mRNA levels by real-time quantitative RT-PCR assay, demonstrating that FoxO $1^{\text {S253A }}$ transgenic mice were associated with significantly higher MTTP mRNA expression when compared with age- and sex-matched control littermates (Figure 4E). These results were consistent with previous observations that FoxO1 ${ }^{\text {S253A }}$ transgenic mice are associated with altered TG metabolism (36).

To corroborate the above findings, we studied molecular association of FoxO1 with the MTP promoter in liver. FoxO1 ${ }^{\text {S253A }}$ transgenic mice were sacrificed after a 16 -h fast. Liver tissues were immediately fixed in $2 \%$ of formaldehyde and subjected to ChIP assay using anti-FoxO1 antibody or preimmune serum. 

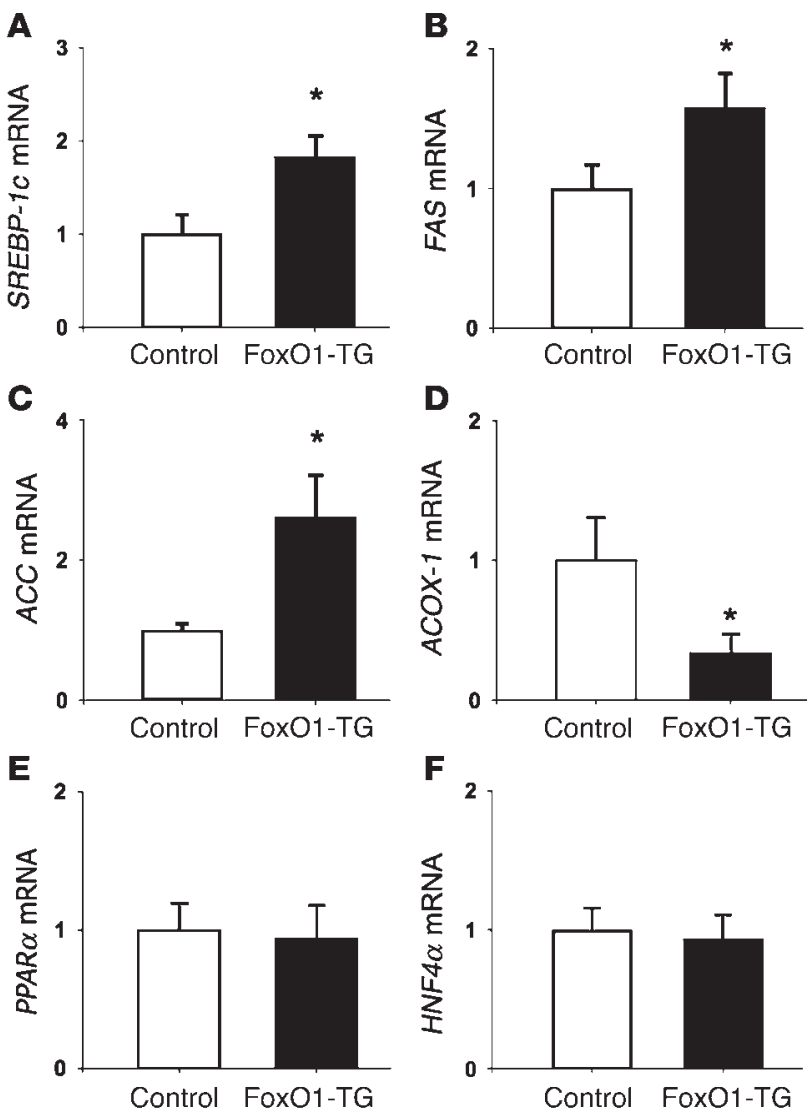

The resulting immunoprecipitates were analyzed by PCR assay using primers flanking the IRE sequence of the MTP promoter. As shown in Figure 4F, specific DNA bands corresponding to the MTP promoter were detected in the immunoprecipitates by anti-FoxO1 antibody. Likewise, specific protein bands corresponding to FoxO1 were detected by immunoblot assay in the immunoprecipitates by anti-FoxO1 antibody (Figure 4G). In contrast, the immunoprecipitates derived from preimmune serum were negative, when analyzed in the same PCR and western blot assays. As a positive control, we subjected aliquots $(1 \mu \mathrm{l})$ of input DNA samples to the same PCR assay. A 500-bp DNA band corresponding to the MTP promoter was detected in all input DNA samples (Figure 4F). As a negative control, we performed PCR analysis using a pair of off-target primers as described above. No specific DNA was produced from the immunoprecipitates by preimmune IgG or anti-FoxO1 antibody (Figure 4F).

To determine the effect of FoxO1 gain-of-function on hepatic TG metabolism, we studied the expression of hepatic genes involved in

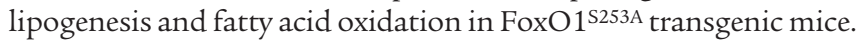
When compared with age- and sex-matched controls, FoxO1 $1^{\text {S253A }}$ transgenic mice displayed significantly higher levels of sterol regulatory element binding protein-1c (SREBP-1c), fatty acid synthase $(F A S)$, and acetyl CoA carboxylase (ACC) mRNA in liver (Figure 5). In contrast, the expression level of acetyl-coA oxidase 1 (ACOX-1), a hepatic enzyme involved in $\beta$-oxidation, was downregulated in FoxO1 ${ }^{\mathrm{S} 253 \mathrm{~A}}$ transgenic mice (Figure 5D). No significant differences in hepatic PPAR $\alpha$ or hepatocyte nuclear factor $4 \alpha$ (HNF4 $\alpha$ ) mRNA levels were detected in FoxO $1^{\mathrm{S} 253 \mathrm{~A}}$ transgenic mice compared with control mice (Figure 5, E and F). While there is evidence that

\section{Figure 5}

Effect of transgenic FoxO1 production on hepatic gene expression. Liver tissues collected from sacrificed mice in 6-month old FoxO1S253A transgenic $(n=6)$ and control groups $(n=6)$ were used for the preparation of total RNA. Hepatic mRNA levels of SREBP-1C (A), FAS (B), ACC (C), ACOX-1 (D), PPAR $\alpha(\mathrm{E})$, and HNF4 $\alpha$ (F) were determined using real-time quantitative RT-PCR assay. ${ }^{\star} P<0.05$ versus control.

hepatic MTP production is also regulated by PPAR $\alpha$ or HNF4 $\alpha$ in mouse livers (37), these data rule out the possibility that the induction of hepatic MTP expression is secondary to altered PPAR $\alpha$ or $\mathrm{HNF} 4 \alpha$ expression in liver with elevated FoxO1 activity.

To strengthen the above results, we transduced HepG2 cells with FoxO1-ADA vector, followed by the quantification of TG concentrations in culture medium. As shown in Figure 6A, FoxO1-ADA production resulted in a marked elevation of TG levels in culture medium. In addition, hepatic apoB100 mRNA and protein levels were significantly increased in response to FoxO1-ADA production in HepG2 cells (Figure 6, B and C). This effect correlated with the induction of MTP expression by FoxO1-ADA in cultured HepG 2 cells (Figure 1) and elevated TG secretion from FoxO1ADA-expressing HepG2 cells (Figure 6A).

To corroborate these results, we subjected FoxO1-ADA-expressing HepG2 cells to labeling with $\left[{ }^{35} \mathrm{~S}\right]$-methionine to study apoB100 secretion. Both conditioned medium and cell lysates were subjected to electrophoresis on 5\% SDS-polyacrylamide gels, which were dried prior to autoradiography. Significantly higher levels of apoB proteins were detected in both conditioned medium and cells that were pretransduced with FoxO1-ADA vector (Figure 6, D and E).

Effect of FoxO1 deficiency on MTP and VLDL production. To study the effect of FoxO1 loss-of-function on hepatic MTP expression and VLDL production, we employed an RNAi-mediated gene silencing approach to downregulate FoxO1 expression in liver. Three plasmids encoding a 19-bp DNA that is complementary to 3 regions of the FoxO1 cDNA sequence (11-29 nt, 814-832 nt, and 1,381-1,399 nt) under the control of the mouse U6 promoter were constructed (Supplemental Figure 1; supplemental material available online with this article; doi:10.1172/JCI32914DS1). Each plasmid construct was tested for its ability to downregulate hepatic FoxO1 expression in mice. We followed an established hydrodynamic DNA transfer protocol to deliver plasmid FoxO1-RNAi DNA into liver of normal C57BL/6J mice $(n=6)$. This approach has been shown to result in transient transgene expression mainly in liver (38). Three days after DNA injection, we determined blood glucose levels under fed and fasting conditions. When compared with control plasmid DNA injected mice, only one FoxO1-RNAi (11-29 nt) was effective in reducing fasting blood glucose levels (Supplemental Figure 2A) without alterations in nonfasting blood glucose levels (Supplemental Figure 2B). In contrast, no significant effects on blood glucose levels were detected under fed and fasting conditions for the other 2 FoxO1-RNAi constructs that are complementary to FoxO1 cDNA 814-833 nt and 1,380-1,399 nt.

After 15-h fasting, mice were sacrificed on day 4 following FoxORNAi DNA administration, and liver tissues were subjected to realtime quantitative RT-PCR analysis. As shown in Supplemental Figure 3A, FoxO1-RNAi (11-29 nt) vector-treated mice displayed significantly reduced FoxO1 mRNA levels in liver. This effect correlated with significantly reduced expression of 2 gluconeogenic genes, namely phosphoenolpyruvate carboxykinase (PEPCK) and glucose-6-phosphatase (G6Pase), in FoxO1-RNAi-treated mice 
A

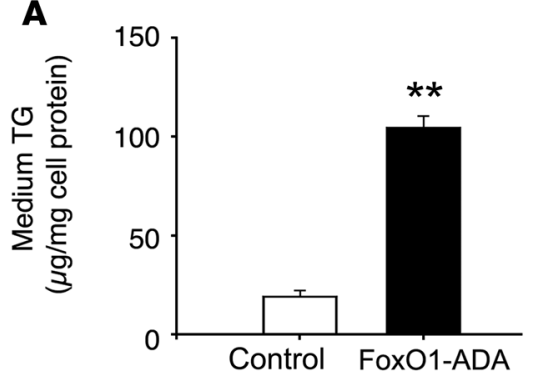

D

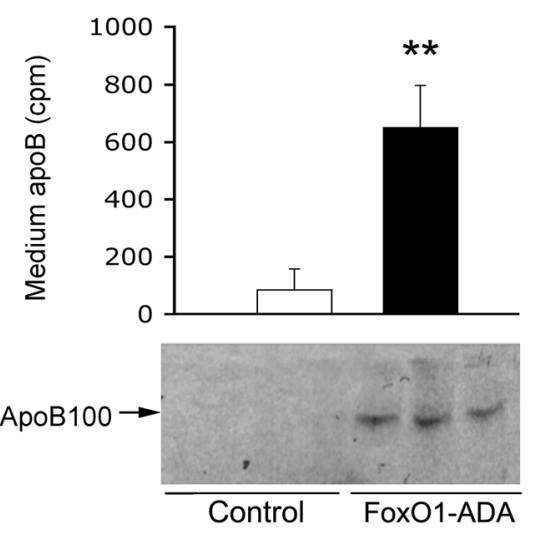

B

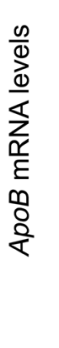

$\mathbf{E}$

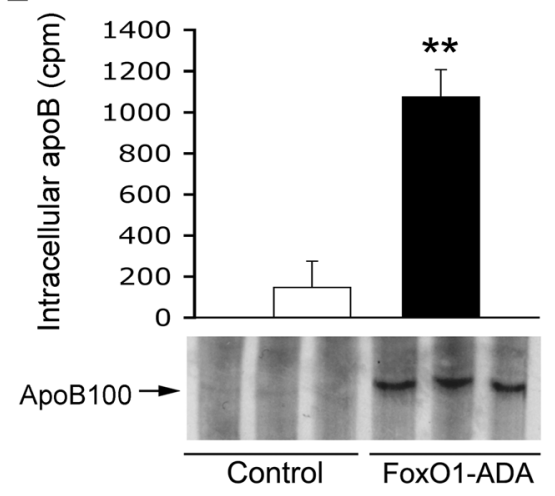

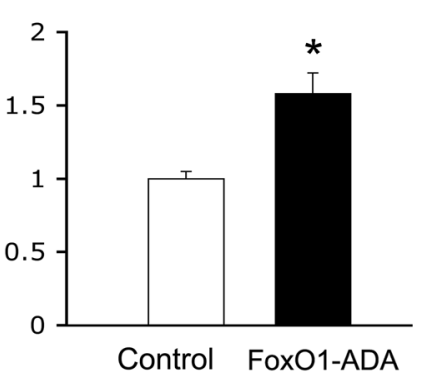

C
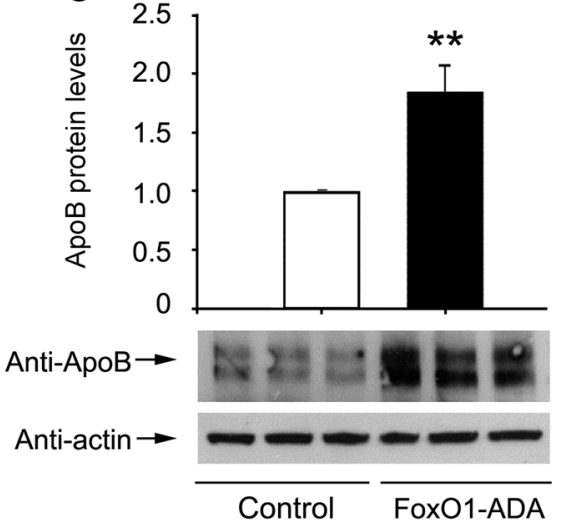

\section{Figure 6}

Effect of FoxO1 on VLDL-TG production in HepG2 cells. HepG2 cells in 6-well plates were transduced with either control or FoxO1-ADA vector at an $\mathrm{MOI}$ of $100 \mathrm{pfu} / \mathrm{cell}$. (A) After 24-h incubation, conditioned media were collected for the determination of TG levels. Cells were collected for the determination of apoB100 mRNA abundance by real-time quantitative RT-PCR assay using $\beta$-actin mRNA as control (B), and for determination of apoB100 protein levels by semiquantitative immunoblot assay using anti-apoB100 and anti-actin antibodies (C). In addition, HepG2 cells pretransduced with Adv-FoxO1-ADA and Adv-null vectors were pulse-labeled with $200 \mu \mathrm{Ci} / \mathrm{ml}$ of L-[35S]-methionine for $1 \mathrm{~h}$, followed by a 30-min chase with $10 \mathrm{mM}$ unlabeled methionine. Conditioned medium and cell lysates were subjected to electrophoresis on 5\% SDS-polyacrylamide gels, which were dried prior to autoradiography. Gel slices corresponding to apoB100 protein bands were excised from gels with the aid of autoradiogram for the determination of radioactive content of apoB100 proteins that were secreted into conditioned medium (D) and present in cells (E). Data were from 3 experiments. ${ }^{*} P<0.05 ;{ }^{* *} P<0.001$ versus control.

(Supplemental Figure 3, B and C). These results are consistent with the idea that FoxO1 plays an important role in hepatic gluconeogenesis. RNAi-mediated FoxO1 deficiency in liver resulted in reduced hepatic gluconeogenesis, contributing to lower fasting blood glucose levels. This study validated the RNAi-mediated approach for generating FoxO1-deficient liver in adult mice.

We constructed an adenovirus expressing FoxO1-RNAi (11-29 nt) under control of the U6 promoter and studied the effect of FoxO1 deficiency on hepatic MTP expression and VLDL production in adult mice. Adv-FoxO1-RNAi and control Adv-null adenoviral vectors were administered to 2 groups of C57BL/6J mice $(n=6)$ at a predefined dose of $1.5 \times 10^{11} \mathrm{pfu} / \mathrm{kg}$, as previously described (33). This vector dose resulted in about $70 \%$ transduction of hepatocytes in a mouse liver, with little transduction in other tissues (33). Three days after vector administration, no differences in nonfasting blood glucose levels were detected between control $(123 \pm 7 \mathrm{mg} / \mathrm{dl})$ and FoxO1-RNAi vector-treated $(120 \pm 4 \mathrm{mg} / \mathrm{dl})$ groups. However, in response to a 24-h fast, FoxO1-RNAi-treated mice exhibited significantly reduced fasting blood glucose levels (Figure 7A), accompanied by a small but insignificant reduction in plasma insulin levels (Figure 7B). Relatively higher plasma cholesterol levels (Figure 7C) were detected in FoxO1-RNAi vector-treated mice. No significant differences in fasting plasma TG
(Figure 7D) or nonesterified fatty acid (NEFA) (Figure 7E) levels were detected in FoxO1-RNAi vector-treated mice compared with control vector-treated mice.

To determine the effect of FoxO1-RNAi on VLDL production, mice were fasted at day 5 after vector administration for $5 \mathrm{~h}$, followed by intravenous injection of tyloxapol $(500 \mathrm{mg} / \mathrm{kg}$ body weight). Aliquots $(25 \mu \mathrm{l})$ of tail vein blood were sampled at different times for the determination of plasma TG levels. As shown in Figure 7F, significantly lower plasma TG levels were detected in the FoxO1-RNAi group at all time points following tyloxapol administration. Based on the slopes of plasma TG profiles, we calculated hepatic VLDL production rates, defined as the amount of TG produced per kg body weight per unit of time. When compared with control mice, FoxO1-RNAi treatment was associated with a $30 \%$ reduction in hepatic VLDL production (Figure $7 \mathrm{G}$ ). Aliquots of plasma ( $20 \mu \mathrm{g}$ proteins) obtained from mice at $80 \mathrm{~min}$ after tyloxapol injection were subjected to immunoblot assay using an anti-apoB antibody. As shown in Figure 7H, significantly reduced plasma apoB levels were detected in the FoxO1-RNAi group.

To correlate VLDL production with hepatic MTP expression, we sacrificed mice after an overnight fast at day 10 after vector administration and determined hepatic TG content. As shown in Figure 7I, FoxO1-RNAi vector-treated mice displayed significantly 
A

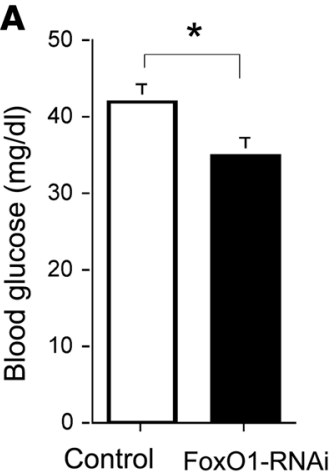

E



I

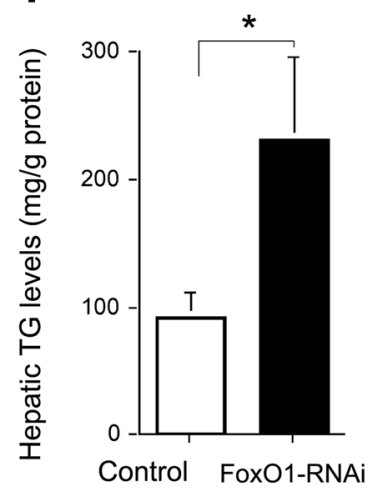

B

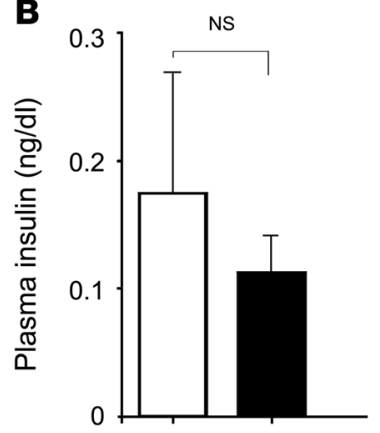

Control FoxO1-RNAi

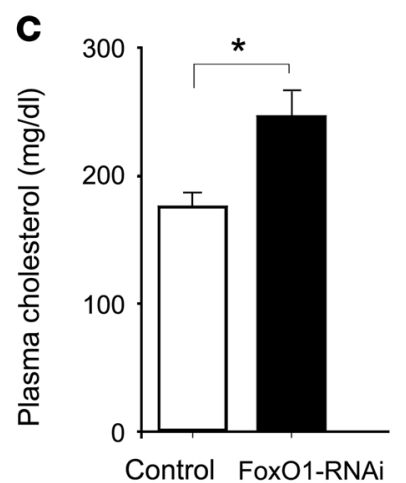

G

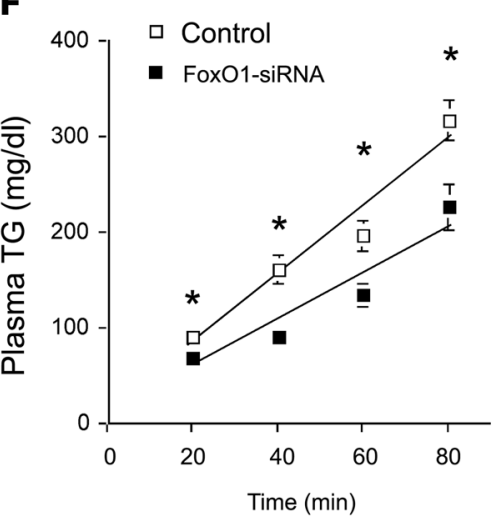

J
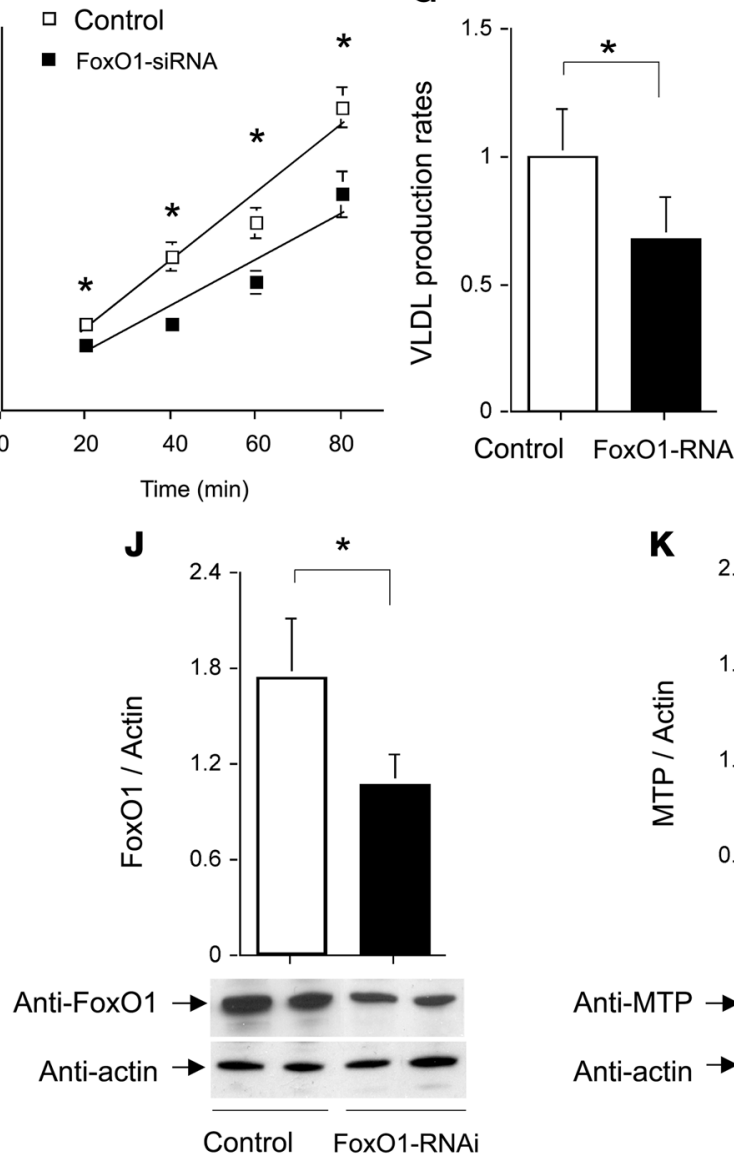

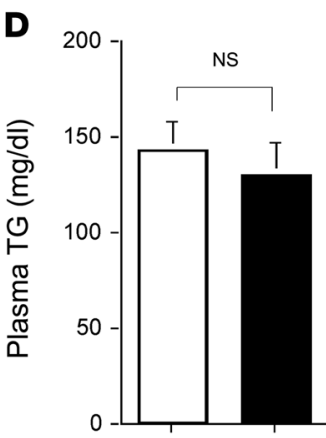

Control FoxO1-RNAi

H

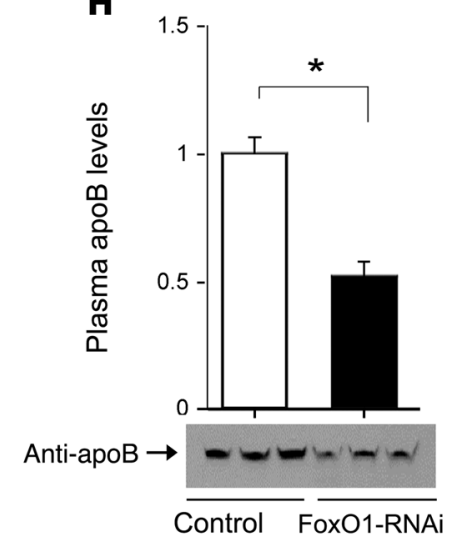

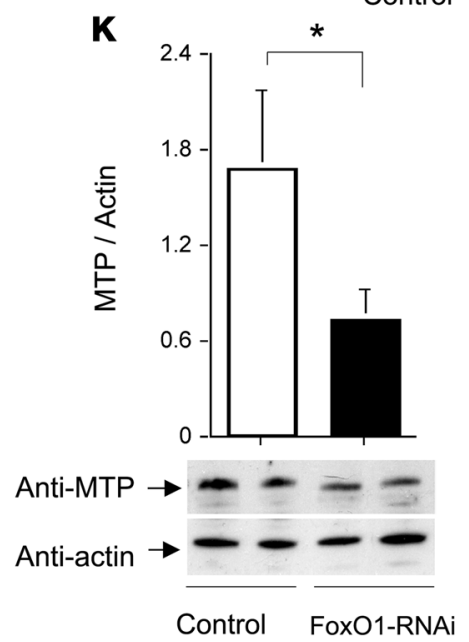

Figure 7

VLDL production in hepatic FoxO1-deficient mice. Male C57BL/6J mice (9 weeks old) were stratified by body weight and randomly assigned to 2 groups $(n=8)$, which were intravenously injected with Adv-FoxO1-RNAi or Adv-null vectors at $1.5 \times 10^{11}$ pfu/kg body weight. Three days after vector administration, mice were fasted for $24 \mathrm{~h}$, followed by the determination of fasting blood glucose (A), plasma insulin (B), plasma cholesterol (C), plasma TG (D), and plasma NEFA levels (E). Mice were intravenously injected with $500 \mathrm{mg} / \mathrm{kg}$ of tyloxapol on day 5 after a 5 -h fast. (F) Plasma TG levels were determined at different times. The relative rates of hepatic VLDL production, defined as mg of TG produced per $\mathrm{kg}$ of body weight per unit of time, were calculated from $\mathbf{F}$ based on the slopes of plasma TG profiles. (G) The mean VLDL production rates were compared between control and FoxO1-RNAi groups. (H) Aliquots of plasma (20 $\mu$ g protein) 80 min after tyloxapol injection were analyzed by semiquantitative western blot assay using anti-apoB antibody for the determination of plasma apoB secretion. Mice were sacrificed after an overnight fast on day 10. (I) Liver tissues were collected for the determination of hepatic TG content. Aliquots of liver tissue were used for the preparation of hepatic protein extracts, which were subjected to semiquantitative immunoblot assay for the determination of hepatic FoxO1 (J) and MTP $(\mathbf{K})$ protein levels in control and FoxO1-RNAi groups, using actin protein as an internal control. ${ }^{*} P<0.05$ versus control. 

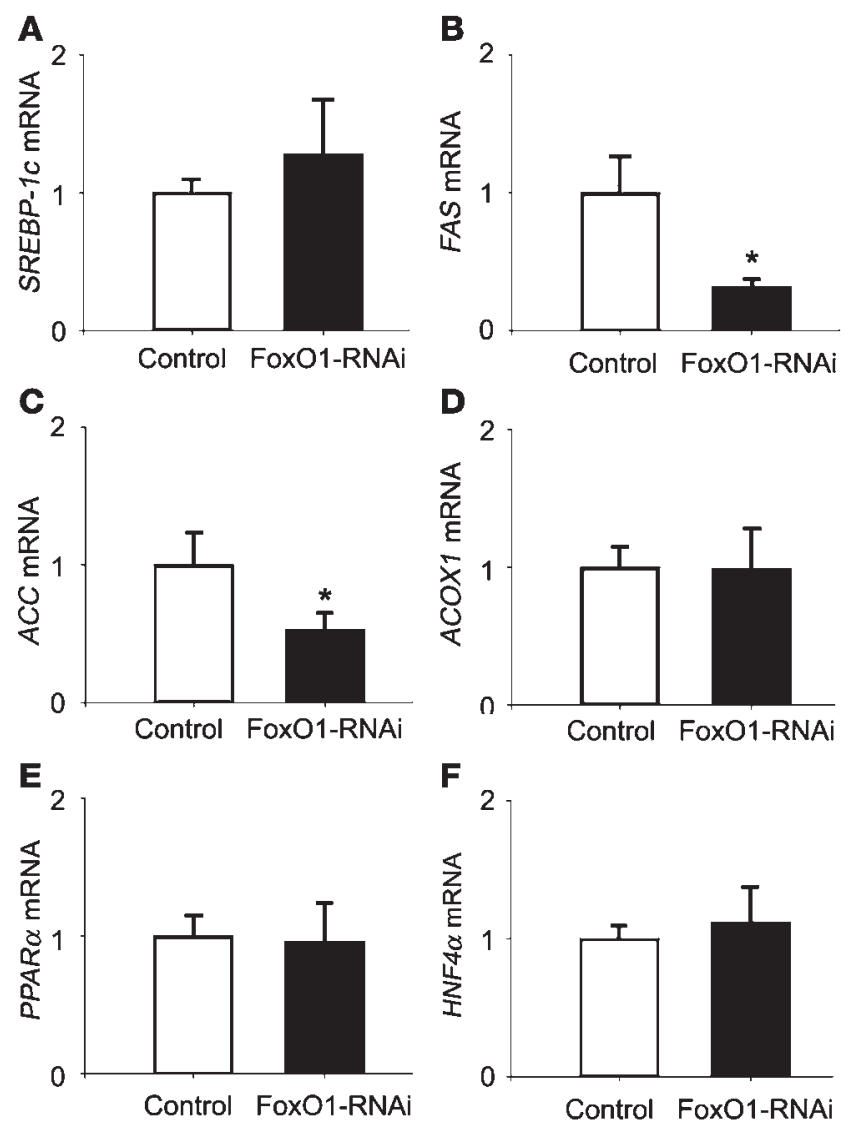

higher TG content, correlating with reduced VLDL production (Figure 7G). In addition, we determined hepatic FoxO1 and MTP levels by semiquantitative immunoblot assay. As shown in Figure 7J, hepatic FoxO1 levels were significantly reduced in response to FoxO1-RNAi-mediated gene silencing in the liver. This effect correlated with significantly lower MTP protein levels in FoxO1-RNAi vector-treated mice (Figure 7K). Thus, RNAi-mediated FoxO1 deficiency in liver resulted in reduced hepatic MTP levels and diminished VLDL production in adult mice.

To determine the effect of FoxO1 loss-of-function on hepatic metabolism, we studied the expression levels of genes involved in hepatic lipogenesis in FoxO1-RNAi vector-treated mice. As shown in Figure 8, FoxO1 loss-of-function resulted in about $40 \%$ reduction in both FAS and ACC mRNA levels, without altering hepatic SREBP-1c mRNA expression. Furthermore, no differences in hepatic expression levels of ACOX1, PPAR $\alpha$, or HNF4 $\alpha$ mRNA were detected between FoxO1-RNAi and control groups.

Hepatic MTP expression in obesity and diabetes. Hepatic VLDL overproduction is considered a contributing factor in the pathogenesis of hypertriglyceridemia associated with obesity and diabetes. To address the pathophysiological significance of FoxO1-mediated induction of MTP production, we determined hepatic MTP levels in high-fat diet-induced obese and diabetic $d b / d b$ mice. Male C57BL/6J mice were induced to obesity $(51 \pm 3.8$ g versus $23 \pm 1.8 \mathrm{~g}$ in regular chow-fed control mice; $P<0.001, n=6$ ) following 8 weeks of high-fat feeding. Obese C57BL/6J mice displayed elevated plasma VLDL-TG levels (Supplemental Figure $4 \mathrm{~A})$, correlating with increased plasma TG levels $(187 \pm 21 \mathrm{mg} / \mathrm{dl}$ versus $120 \pm 8 \mathrm{mg} / \mathrm{dl}$ in lean control mice; $P<0.01$ by ANOVA).

\section{Figure 8}

Effect of RNAi-mediated FoxO1 knockdown on hepatic gene expression. Liver tissues collected from sacrificed C57BL/6J mice in control and RNAi treatment groups ( $n=8$ per group) were used for the preparation of total RNA. Hepatic mRNA levels of SREBP-1C (A), FAS (B), ACC (C), ACOX-1 (D), PPAR $\alpha(\mathbf{E})$, and $H N F 4 \alpha(\mathbf{F})$ were determined using real-time quantitative RT-PCR. ${ }^{\star} P<0.05$ versus control.

In addition, obese C57BL/6J mice exhibited significantly elevated total plasma cholesterol levels $(138 \pm 9 \mathrm{mg} / \mathrm{dl}$ versus $85 \pm 5 \mathrm{mg} / \mathrm{dl}$ in lean control mice; $P<0.01$ by ANOVA), culminating in markedly increased HDL cholesterol levels (Supplemental Figure 4B). Using semiquantitative immunoblot assay, we detected a significant elevation in hepatic MTP levels in obese C57BL/6J mice (Supplemental Figure 4C). This effect paralleled the induction of FoxO1 production and nuclear localization in liver in response to high-fat feeding (39).

Likewise, we determined plasma lipoprotein profiles of diabetic $d b / d b$ mice. When compared with heterozygous $d b /+$ control littermates (mean body weight, $25.2 \pm 0.6 \mathrm{~g} ; 6$ months old, $n=6$ ), diabetic $d b / d b$ mice (mean body weight, $51.1 \pm 1.52 \mathrm{~g} ; n=6$ ) exhibited significantly higher plasma levels of TG (Supplemental Figure 4D) and total cholesterol (Supplemental Figure 4E). In accordance with altered lipid metabolism, diabetic $d b / d b$ mice exhibited significantly increased MTP production in liver (Supplemental Figure $4 \mathrm{~F}$ ). This effect is concomitant with augmented FoxO1 production along with its skewed subcellular distribution to the nucleus in livers of diabetic $d b / d b$ mice $(33,36)$ (Supplemental Figure 5).

RNAi-mediated FoxO1 knockdown in diabetic db/db mice. To investigate the effect of FoxO1 deficiency on hepatic MTP expression, we delivered FoxO1-RNAi vector into the liver of obese $d b / d b$ mice using a scrambled RNAi vector as a control. As described above, this approach generated a liver-specific FoxO1-deficient model in adult mice. Hepatic FoxO1-RNAi production resulted in a significant reduction in fasting blood glucose levels (Figure 9A), accompanied by decreased fasting plasma insulin levels in $d b / d b$ mice (Figure 9B). In contrast, hyperglycemia along with hyperinsulinemia persisted in $d b / d b$ mice receiving the control scrambled RNAi vector (Figure 9, A and B). These data were in line with our previous observations that hepatic production of FoxO1 dominant-negative mutant resulted in significantly improved fasting blood glucose profiles along with a significant reduction in fasting plasma insulin levels in diabetic $d b / d b$ mice (33). Five days following RNAi vector administration, we injected tyloxapol intravenously into individual mice to block plasma VLDL-TG hydrolysis and clearance, followed by the determination of hepatic VLDLTG production. RNAi-mediated FoxO1 knockdown resulted in significantly reduced VLDL-TG production (Figure 9, C and D), correlating with the reduction of plasma apoB secretion in FoxO1-RNAi vector-treated $d b / d b$ mice (Figure 9E). Plasma TG levels remained unchanged in FoxO1-RNAi when compared with scrambled RNAi vector-treated $d b / d b$ mice (Figure 9F). Instead, we detected significant increases in plasma NEFA levels (Figure 9G) and cholesterol levels (Figure 9H) in FoxO1-RNAi vectortreated $d b / d b$ mice. No differences in body weight were detected (Figure 9I), precluding the possibility that the observed reduction in hepatic VLDL-TG production and apoB secretion was secondary to body weight changes in FoxO1-RNAi vector-treated $d b / d b$ mice. After 1 week of hepatic FoxO1-RNAi expression, mice were sacrificed and liver tissues were subjected to immunoblot assay. 
A

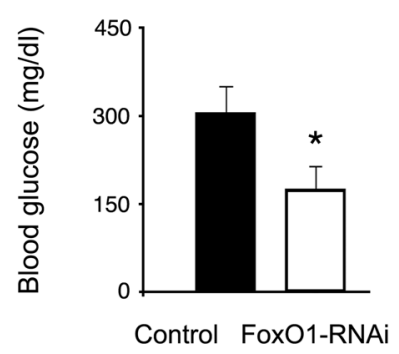

E



I

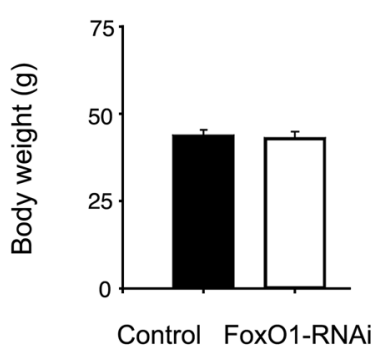

J



B

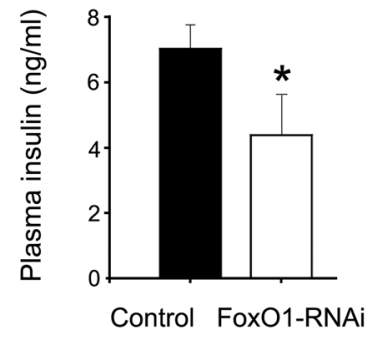

F

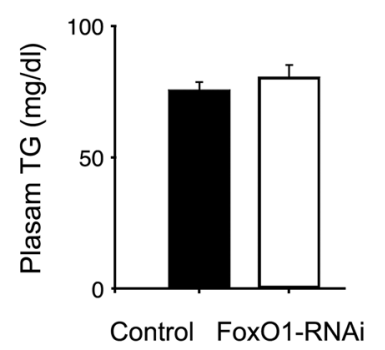

$\mathbf{K}$



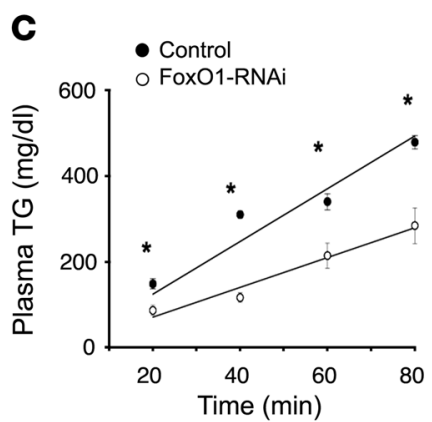

G

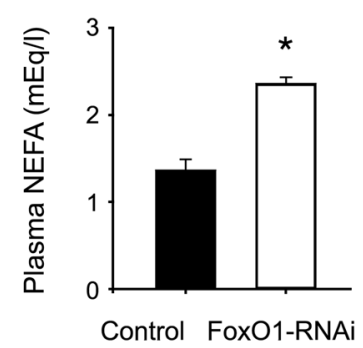

D

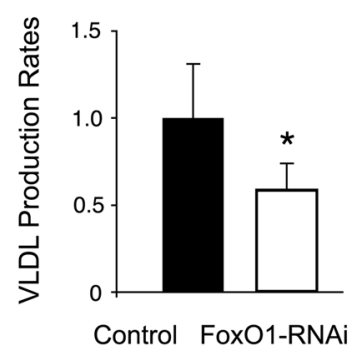

H

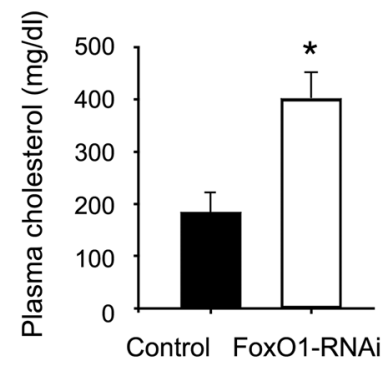

L

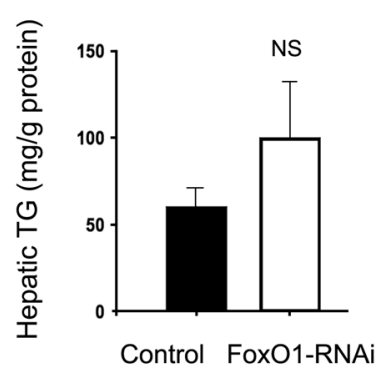

\section{Figure 9}

Effect of FoxO1 knockdown on hepatic metabolism in $d b / d b$ mice. Male $d b / d b$ mice at 3 months of age were stratified by body weight and randomly assigned to 2 groups $(n=8)$, which were intravenously injected with FoxO1-RNAi or scrambled RNAi vector at $1.5 \times 10^{11}$ pfu/kg body weight. Three days after vector administration, mice were fasted for $24 \mathrm{~h}$ for the determination of fasting blood glucose (A) and plasma insulin levels (B). Mice were intravenously injected with $500 \mathrm{mg} / \mathrm{kg}$ of tyloxapol on day 5 after a 5 -h fast. (C) Plasma TG levels were determined at different times. (D) The relative rates of hepatic VLDL production were calculated based on the slopes of plasma TG profiles from $\mathbf{C}$ and compared between control scrambled RNAi and FoxO1-RNAi groups. (E) Aliquots of plasma (20 $\mu \mathrm{g}$ protein) 80 min after tyloxapol injection were analyzed by semiquantitative western blot assay using anti-apoB antibody for the determination of plasma apoB secretion. Fasting plasma levels of TG $(\mathbf{F})$, NEFA $(\mathbf{G})$, and cholesterol $(\mathbf{H})$ were determined at day 3 after vector administration. (I) Body weight was determined on day 7 . Mice were sacrificed on day 7 , and liver tissues were collected for the determination of hepatic protein levels of FoxO1 (J) and MTP (K) as well as hepatic TG content (L). ${ }^{*} P<0.05$ versus control.

We detected a 70\% reduction in hepatic FoxO1 levels (Figure 9J), accompanied by a concomitant reduction in hepatic MTP protein levels (Figure 9K) in FoxO1-RNAi vector-treated mice. Although an induction in hepatic TG levels was detected in FoxO1-RNAi vector-treated $d b / d b$ mice, the degree of induction did not reach a significant level when compared with scrambled RNAi vectortreated $d b / d b$ mice (Figure 9L).

RNAi-mediated FoxO1 knockdown in FoxO1 ${ }^{\text {S253A }}$ transgenic mice. To further address the physiological significance of FoxO1mediated regulation of hepatic MTP and VLDL-TG production, we delivered FoxO1-RNAi vector into livers of FoxO1 ${ }^{\text {s253A }}$ transgenic mice. As shown in Figure 10A, hepatic FoxO1-RNAi expression resulted in significant reduction in fasting blood glucose levels, correlating with the reduction of hepatic FoxO1 levels in FoxO1-RNAi vector-treated mice (Figure 10B). However, no significant differences in fasting plasma insulin levels were detected in FoxO1-RNAi versus scrambled RNAi vector-treated mice, although plasma insulin levels were reduced to basal levels after 16-h fasting (Figure 10C). After 5 days of hepatic FoxO1RNAi expression, we administered tyloxapol intravenously into both FoxO1-RNAi and scrambled RNAi vector-treated mice. As shown in Figure 10D, significantly lower plasma TG levels were 
A



E

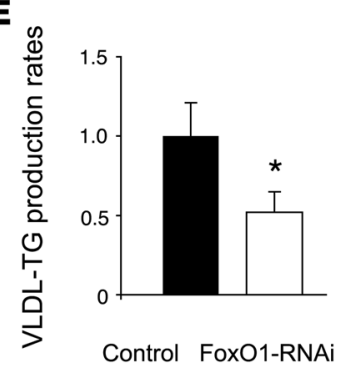

I

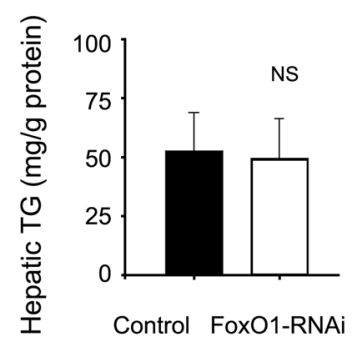

B

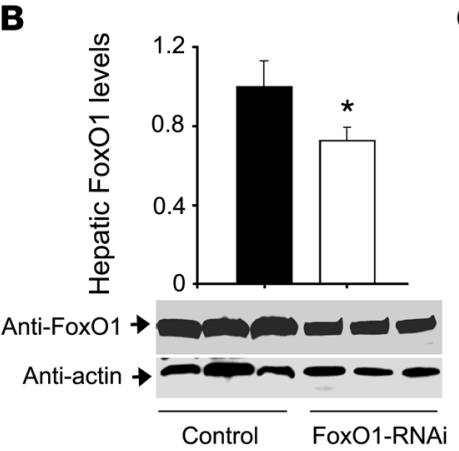

$\mathbf{F}$

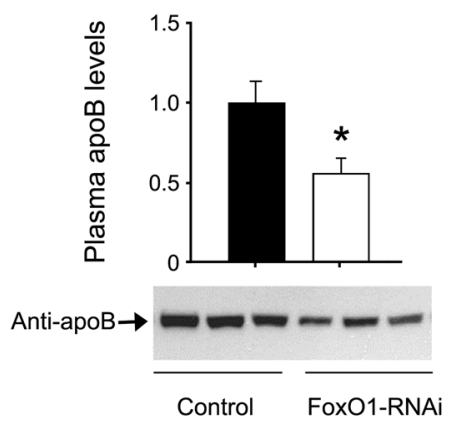

J

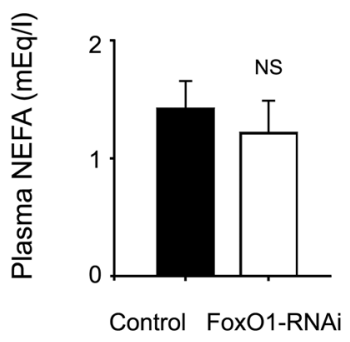

C

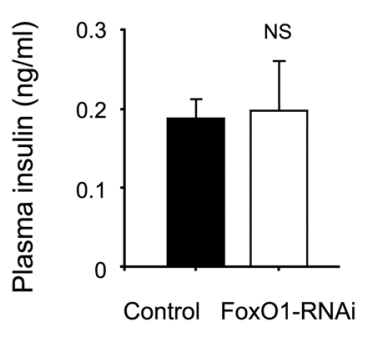

G



K

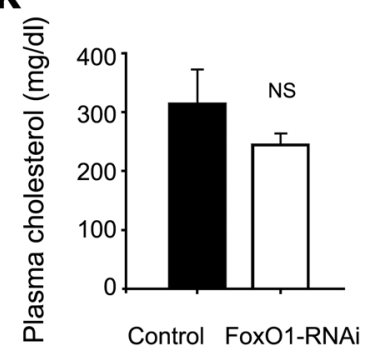

D

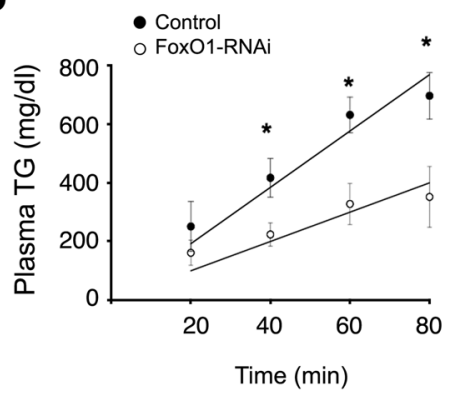

H

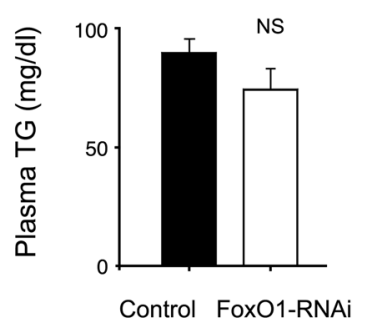

L

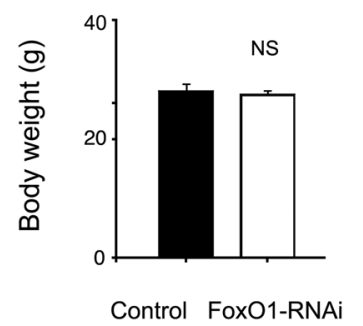

Figure 10

Effect of FoxO1 knockdown on hepatic metabolism in FoxO1 transgenic mice. Male FoxO1S253A transgenic mice at 4 months of age were stratified by body weight and randomly assigned to 2 groups $(n=8)$, which were intravenously injected with FoxO1-RNAi or scrambled RNAi vector at $1.5 \times 10^{11} \mathrm{pfu} / \mathrm{kg}$ body weight. (A) Fasting blood glucose levels. (B) Hepatic FoxO1 protein levels. (C) Fasting plasma insulin levels. (D) Plasma TG levels after intravenous administration of tyloxapol. (E) Hepatic VLDL production rates. (F) Plasma apoB secretion. Aliquots of plasma (20 $\mu \mathrm{g}$ protein) 80 min after tyloxapol injection were analyzed by semiquantitative western blot assay using anti-apoB antibody. (G) Hepatic MTP protein levels. (H) Fasting plasma TG levels. (I) Hepatic TG levels. (J) Fasting plasma NEFA levels. (K) Fasting plasma cholesterol levels. (L) Body weight. All data were obtained between 4 and 8 days from mice fasted for $24 \mathrm{~h}$ after vector administration. ${ }^{*} P<0.05$ versus control.

observed at all time points following tyloxapol infusion, indicating that FoxO1 loss-of-function was associated with reduced VLDL-TG production in FoxO1 ${ }^{\mathrm{S} 253 \mathrm{~A}}$ transgenic mice (Figure 10E). This effect correlated with the reduction in plasma apoB secretion following tyloxapol injection (Figure 10F) and reduced hepatic MTP levels in FoxO1-RNAi vector-treated mice (Figure 10G). Consistent with data obtained in $d b / d b$ mice, FoxO1-RNAi expression did not significantly alter fasting plasma TG levels (Figure 10H) and hepatic TG levels (Figure 10I). Likewise, no significant differences were detected in plasma levels of NEFA (Figure 10J) and total cholesterol (Figure 10K) between FoxO1RNAi and control groups. The mean body weight in both FoxO1RNAi and scrambled RNAi groups remained unchanged (Figure $10 \mathrm{~L}$ ), ruling out the possibility that the observed reduction in hepatic MTP expression and VLDL-TG production was due to body weight changes in FoxO1-RNAi vector-treated mice.

\section{Discussion}

Our goals in this study were 2-fold: to better understand the mechanism by which insulin regulates VLDL assembly and production in liver, and to gain insight into the linkage of hepatic insulin resistance to VLDL overproduction in the pathogenesis of hypertriglyceridemia. Using gain-of-function versus loss-of-function approaches, we investigated the effect of FoxO1 on hepatic regulation of MTP, a molecular chaperone that resides in the ER lumen and catalyzes the rate-limiting step in VLDL assembly and production in liver. We show that hepatic MTP production was stimulated by FoxO1 and inhibited by insulin. This was consistent with the ability of insulin to promote FoxO1 phosphorylation and translocation from the nucleus to the cytoplasm $(29,40)$. FoxO1-ADA also stimulated MTP production in HepG2 cells, but its stimulatory effect was refractory to insulin inhibition due to the inability of FoxO1-ADA to undergo insulin-dependent phos- 


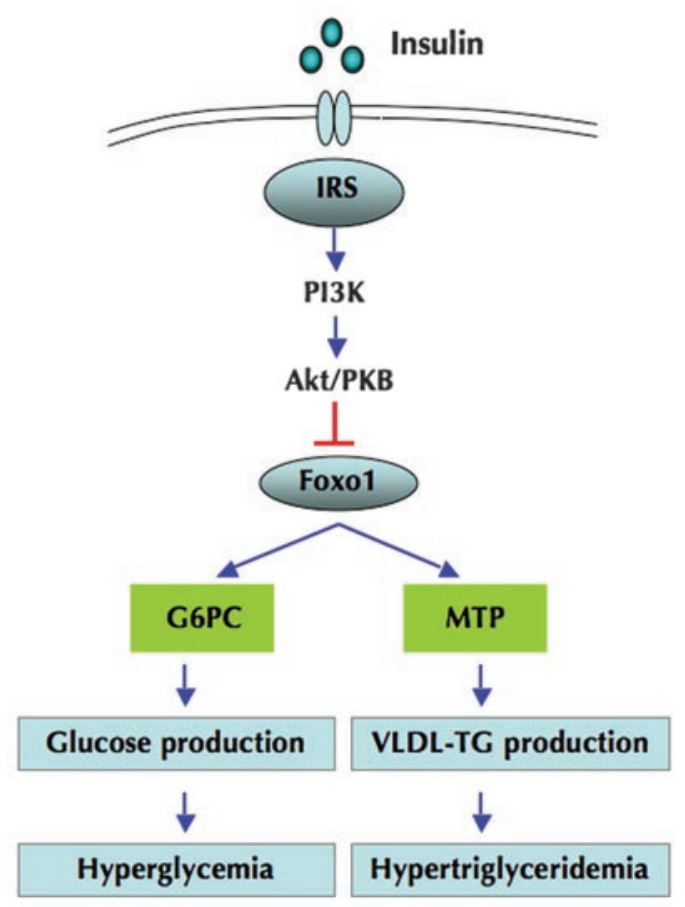

phorylation and nuclear exclusion $(31,41)$. FoxO1 was shown to physically bind to the MTP promoter and functionally mediate the inhibitory effect of insulin on MTP expression. FoxO1 transgenic expression resulted in increased MTP production in liver, contributing to augmented hepatic VLDL production and elevated plasma TG levels in mice. Conversely, FoxO1 loss-of-function suppressed hepatic MTP expression, resulting in reduced hepatic VLDL production. Furthermore, hepatic MTP levels were increased, coinciding with increased FoxO1 nuclear localization in mice with altered plasma TG metabolism (36). Together these results implicate the MTTP gene as a FoxO1 target, suggesting that FoxO1 mediates insulin action on hepatic MTP expression in modulating hepatic VLDL production and plasma TG metabolism.

What is the physiological significance of these findings? It is known that hepatic VLDL production depends on substrate availability, which is sensitive to insulin inhibition $(7,42-44)$. Such an inhibitory effect has been viewed as an acute mechanism to prime liver for rapid adaptation to metabolic shift from fasting to fed states. First, this mechanism acts to limit postprandial excursion of plasma TG profiles by curbing continuous hepatic VLDL production after meals. Second, it helps preserve hepatic TG stores for post-absorptive VLDL-TG secretion in response to reduced insulin action between meals or during fasting. Nevertheless, a unifying idea regarding the inhibitory effect of insulin on hepatic VLDL production is lacking. One potential mechanism is that insulin inhibits VLDL assembly and secretion via a direct mechanism by promoting apoB degradation. In keeping with this notion, insulin is shown to suppress apoB expression and promote apoB degradation in cultured primary rat hepatocytes, HepG 2 cells, and perfused rat livers (45-51). An alternative mechanism is that insulin inhibits hepatic VLDL output via an indirect mechanism by limiting its substrate FFA availability in the circulation (52). This idea is consistent with the ability of insulin to restrain FFA mobilization from adipose tissue (53), but is at variance with clinical evidence that insulin acutely

\section{Figure 11}

Insulin signaling through FoxO1 regulates hepatic glucose and VLDLTG production. Insulin inhibits FoxO1 activity via Akt/PKB-dependent phosphorylation, resulting in FoxO1 nuclear exclusion. This effect is instrumental for liver to curb hepatic glucose and VLDL-TG production and limit postprandial glucose and lipid excursion. Loss of insulin inhibition of FoxO1 activity in insulin-resistant livers results in excessive production of both glucose and VLDL-TG, contributing to the dual pathogenesis of hyperglycemia and hypertriglyceridemia in diabetes.

inhibits hepatic VLDL output and apoB production independent of FFA availability $(54,55)$. In this context, our present studies significantly advance the concept by showing that insulin signaling through FoxO1 is instrumental for insulin-dependent inhibition of hepatic MTP and apoB production in regulating hepatic VLDL assembly and production in liver. Intriguingly, this effect is akin to the mechanism by which insulin suppresses hepatic glucose production and prevents postprandial glucose excursion through the inhibition of PEPCK and G6Pase expression $(29,40)$.

Excessive VLDL production is attributable to the pathogenesis of hypertriglyceridemia. It has been suggested that this effect results from an impaired ability of insulin to curb hepatic VLDL assembly and secretion in the presence of increased FFA infiltration into livers in obesity and type 2 diabetes $(3,4)$. However, factors that link impaired insulin action to unrestrained VLDL production in liver remain elusive. Recent studies by Wolfrum and Stoffel show that hepatic VLDL production is regulated by Foxa2, another member of the forkhead superfamily (56). Foxa2 in complex with its coactivator PPAR $\gamma$ coactivator-1 $\beta$ (PGC-1 $\beta$ ) promotes hepatic MTP expression, contributing to increased VLDL output from liver (57). In response to insulin, Foxa 2 becomes phosphorylated, resulting in its dissociation from PGC-1 $\beta$ and contributing to the inhibition of hepatic VLDL secretion (57). This model, which is apparently consistent with the convergence of Foxa 2 and PGC-1 $\beta$ in mediating hepatic insulin action on hepatic lipid metabolism (58), falls short of reconciling the phenotype of elevated plasma TG levels with the cytosolic localization of Foxa 2 in insulin-resistant states (56). There are studies showing that although Foxa2 binds to the promoter of several hepatic genes, including PEPCK, G6Pase, insulin-like growth factor binding protein 1 (IGFBP-1), and tyrosine aminotransferase (TAT), its binding does not confer insulin responsiveness to target gene expression (59-63). Consistent with these observations, Zhang et al. (64) have shown that Foxa2 does not alter its subcellular localization, as Foxa2 remains constitutively nuclear when the liver undergoes a metabolic shift from fed to fasting states. These studies underscore the importance of Foxa 2 in integrating the transcriptional response of hepatocytes to fasting, but not in modulating target gene expression in response to insulin $(64,65)$.

This has led to the postulation that other factors are involved in regulating hepatic VLDL-TG production in response to insulin. Our studies provide evidence that one such factor is FoxO1. Due to its inability to undergo insulin-dependent phosphorylation and nuclear exclusion, FoxO1 is concentrated in the nucleus of hepatocytes in insulin-resistant states. Our interpretation is that an increased nuclear localization of FoxO1 contributes to its enhanced transcriptional activity in insulin-resistant livers, which serves as a driving force for promoting MTP and VLDL production. In support of this interpretation, we show that hepatic FoxO1 expression levels are increased and this increased FoxO 1 is predominantly localized in the nucleus, correlating with augmented 
MTP production and elevated plasma VLDL-TG levels in high-fat diet-induced obese mice, diabetic $d b / d b$ mice, and high-fructose diet-induced hypertriglyceridemic hamsters $(33,36,39,66)$. In accordance with these findings, several independent studies have reported a positive correlation between increased MTP expression and abnormal TG metabolism in $o b / o b$ mice, non-diabetic obese Zucker rats, and high-fructose diet-fed hamsters (4, 20, 21, 67). Together these data underscore the importance of FoxO1 in mediating insulin action on VLDL-TG metabolism and forebode a close association between the inability of insulin to keep FoxO1 activity in check and unrestrained VLDL production, resulting in undue hypertriglyceridemia in insulin-resistant subjects.

Despite the reduction of hepatic VLDL-TG production, fasting plasma TG levels were not significantly reduced in FoxO1-RNAi vector-treated mice. Instead, we detected a significant reduction in plasma TG levels when plasma VLDL-TG hydrolysis and clearance was blocked by tyloxapol in FoxO1-RNAi-treated mice. These results were reproduced in all 3 models (C57BL/6J, $d b / d b$, and FoxO1 ${ }^{\text {S253A }}$ transgenic mice) following FoxO1-RNAi vector administration. It is plausible that RNAi-mediated FoxO1 knockdown in liver inadvertently affected other hepatic factors that are involved in VLDL-TG catabolism. Further studies are warranted to better understand the underlying physiology of plasma TG metabolism in FoxO1-deficient mice.

It is noteworthy that hepatic levels of apoB100, along with its secretion, were upregulated in response to FoxO1 production, correlating with increased VLDL production in HepG2 cells. ApoB100 is a secretory protein whose lipidation plays a pivotal role in VLDL assembly. It is thought that apoB production is kinetically coupled with its lipidation by MTP, which is tightly regulated by substrate availability at the posttranscriptional level (68). It is possible that the observed increase in apoB100 levels resulted from FoxO1mediated induction of apoB100 expression in concert with the induction of MTP. Further research is needed to address whether FoxO1 targets hepatic apoB gene for trans activation in modulating VLDL-TG metabolism.

In addition to its effect on hepatic VLDL-TG production, increased FoxO1 activity resulting from transgenic FoxO1 expression or adenovirus-mediated FoxO1 production in liver contributed to augmented expression of lipogenic genes including SREBP-1c, $F A S$, and ACC. Similar results were reported in FoxO1-ADA vector-treated mice by Matsumoto et al. (69). This effect, along with increased PGC-1 $\beta$ expression, accounted in part for increased fat deposition in livers of FoxO1-overexpressing mice $(39,69)$. In accordance with these observations, Valenti et al. have shown that increased hepatic FoxO1 activity is associated with nonalcoholic steatohepatitis in humans (70). However, these results seem at variance with $Z$ hang et al. (71), who reported that hepatic SREBP-1C mRNA expression was reduced by $50 \%$ in FoxO1-AAA transgenic mice. It is noted that the FoxO1 transgenic mice used by Zhang et al. were generated on an FVB/N genetic background (71), whereas our transgenic mice were on a C57BL/6J background. Their transgenic mice were fed a high-carbohydrate diet containing $65 \%$ sucrose, in comparison with the regular chow used in our study. Zhang et al. studied the expression of SREBP-1c $6 \mathrm{~h}$ after feeding (71), when lipogenesis is relatively higher, in comparison with our experimental setting, in which SREBP-1c expression was determined after a 16 -h fast. Furthermore, our FoxO ${ }^{\text {s253A }}$ transgenic mice expressed FoxO1 at levels that were 2- to 3-fold higher than control littermates (35). This level is equivalent to hepatic
FoxO1 protein levels detected in high-fat diet-induced obese mice (39) and diabetic $d b / d b$ mice (36). In contrast, only a $50 \%$ increase in hepatic FoxO1 levels was detected in their transgenic mice (71). Together these differences may contribute to the discrepancy in the effect of FoxO1 on lipogenic SREBP-1c expression between our data and the studies by Zhang et al. (71).

Biddinger et al. (72) studied the effect of blunted insulin signaling in liver on hepatic lipid metabolism in liver-specific insulin receptor knockout (LIRKO) mice. LIRKO mice exhibited insulin resistance and hyperinsulinemia, accompanied by dyslipidemia resulting from increased secretion and decreased clearance of apoB-containing lipoproteins. As a result, LIRKO mice developed atherosclerosis when fed an atherogenic diet. However, LIRKO mice had relatively lower plasma TG levels due to reduced hepatic TG synthesis and secretion. This effect correlated with decreased expression of lipogenic genes such as SREBP-1c and FAS in LIRKO mice. Hepatic TG content remained unchanged in LIRKO mice. These results indicate that ablation of insulin signaling in liver is sufficient to produce dyslipidemia and susceptibility to atherosclerosis resulting from augmented apoB secretion in LIRKO mice (72). Although FoxO1 expression was not examined, it is anticipated that FoxO1 activity is upregulated due to the inability of insulin to promote FoxO1 phosphorylation and nuclear exclusion in the liver of LIRKO mice. This raises the hypothesis that FoxO1 knockdown would curb excessive apoB secretion and ameliorate dyslipidemia in LIRKO mice.

While impaired insulin signaling is associated with abnormal metabolism, recent studies by 2 independent groups indicate that hepatic hypersensitivity to insulin resulting from liver-specific depletion of phosphatase and tension homolog deleted on chromosome 10 (PTEN) contributes to disorders in hepatic metabolism $(73,74)$. As PTEN is a negative regulator of the PI3K/Akt pathway, hepatic depletion of PTEN results in enhanced insulin action, accounting for increased glycogen synthesis and augmented lipogenesis in liver at the expense of reduced total body fat. This skewed fat redistribution from other tissues to liver results in hepatomegaly, steatohepatitis, and hepatocellular carcinomas in PTEN knockout mice. Liver-specific deletion of PTEN also results in elevated hepatic TG secretion secondary to increased de novo lipogenesis. These results demonstrate that insulin hypersensitivity causes metabolic abnormalities via a mechanism that is fundamentally different from the metabolic syndrome caused by insulin resistance in obesity and diabetes. PTEN-knockout mice provide a conditional model for studying insulin-target gene expression and hepatic metabolism in insulin-hypersensitive states.

In conclusion, we show that FoxO1 mediates insulin action on hepatic MTP expression in regulating VLDL production and TG metabolism. This conclusion bears 2 lines of significance. First, this model helps prime liver to undergo rapid adaptation to metabolic shift from fasting to refeeding. In response to postprandial insulin release, FoxO1 is phosphorylated and excluded from the nucleus, resulting in the inhibition of hepatic MTP expression. This effect acts to abate VLDL production and limit postprandial TG excursion. Second, in response to impaired insulin action, FoxO1 is translocated into the nucleus, contributing to its enhanced activity in driving hepatic MTP expression and promoting VLDL production. An impaired ability of insulin to check FoxO1 activity may be a causative factor for VLDL overproduction in insulin-resistant liver, accounting for the pathogenesis of hypertriglyceridemia in obesity and type 2 diabetes. As shown in Figure 11, hepatic insulin 
signaling bifurcates at FoxO1 to target genes in glucose and TG metabolism for controlling hepatic glucose production and VLDL secretion, an effect that is critical for maintaining blood glucose and TG levels within the physiological range, as liver undergoes a metabolic shift from fed to fasting states. This effect accounts in part for the underlying mechanism of concomitant perturbations in glucose and lipid metabolism with concurrent manifestations of both hyperglycemia and hypertriglyceridemia, when insulin signaling goes awry in subjects with metabolic syndrome.

\section{Methods}

Cell culture and adenovirus transduction. HepG2 cells were purchased from ATCC, cultured, and transduced with adenoviruses as previously described (36). The adenoviral vectors used were as follows: Adv-CMV-FoxO1 expressing wild-type FoxO1 $\left(1.0 \times 10^{11} \mathrm{pfu} / \mathrm{ml}\right)$, Adv-CMV-FoxO1-ADA expressing constitutively active FoxO1-ADA allele $\left(1.0 \times 10^{11} \mathrm{pfu} / \mathrm{ml}\right)$, AdvLacZ expressing the $\beta$-gal gene $\left(1.25 \times 10^{11} \mathrm{pfu} / \mathrm{ml}\right)$, and the null adenovirus Adv-null $\left(1.25 \times 10^{11} \mathrm{pfu} / \mathrm{ml}\right)$. Adv-FoxO1-RNAi vector was constructed based on the Block-iT Adenoviral RNAi Expression System (Invitrogen). This FoxO1-RNAi vector encodes a 19-bp DNA (5'-CGCCCCAGGTGGTGGAGAC-3') that is complementary to the FoxO1 mRNA sequence (10-29 nt) under the control of mouse U6 promoter. Likewise, a control adenovirus containing the scrambled RNAi (5'-GGACTCGGGCCACCGGGTA-3') under the control of mouse U6 promoter was constructed. Adv-CA-Akt encodes a constitutively active form of Akt, as previously described (75). This constitutively active form of Akt contains the $\mathrm{NH}_{2}$ terminal c-Src myristoylation signal fused to Akt, which targets Akt to the plasma membrane, where it is phosphorylated, resulting in its constitutive activation in cells (76). All adenoviral vectors were produced in HEK293 cells and purified as described in ref. 33. The PI3K activator IRS-1 (Y608) peptide (KKHTDDGYMPMSPGVA) was obtained from EMD Chemicals. The tyrosine-phosphorylated IRS-1 (Y608) peptide binds to the PI3K SH2 domain and activates PI3K, as reported in refs. 77-79. PI3K inhibitor LY294002 (BIOMOL International) has been previously described (80).

Animal studies. C57BL/6J, $d b / d b$, and heterozygous $d b /+$ mice at $8 \mathrm{wk}$ of age were purchased from The Jackson Laboratory. FoxO1 ${ }^{\text {S253A }}$ transgenic mice, obtained from Domenico Accili's lab (Columbia University College of Physicians and Surgeons, New York, New York, USA), were bred at the animal facility of the Children's Hospital of Pittsburgh. To induce obesity, male C57BL/6J mice were fed a high-fat diet (fat content $>60 \mathrm{kcal} \%$, D12491; Research Diets) for 8 weeks. For blood chemistry, mice were fasted for $16 \mathrm{~h}$ and tail vein blood samples were collected for the determination of blood glucose, plasma TG, and cholesterol levels, as previously described (36). All procedures were approved by the IACUC of the Children's Hospital of Pittsburgh.

Hepatic VLDL-TG production assay. Mice were fasted for $5 \mathrm{~h}$, followed by intravenous injection of tyloxapol (Sigma-Aldrich) at $500 \mathrm{mg} / \mathrm{kg}$ body weight. Aliquots of tail vein blood were taken at different times for plasma TG determination as previously described (36).

$V L D L$-apoB secretion assay. HepG2 cells in 6-well microplates were transduced with Adv-FoxO1-ADA and Adv-null vectors (MOI, $100 \mathrm{pfu} / \mathrm{cell}$ ) in MEM supplemented with $10 \%$ FBS. After 24-h incubation, cells were labeled with $200 \mu \mathrm{Ci} / \mathrm{ml}$ of $\mathrm{L}-\left[{ }^{35} \mathrm{~S}\right]$-methionine $(1,175 \mathrm{Ci} / \mathrm{mmol}$; Perkin-Elmer) for $1 \mathrm{~h}$ in DMEM medium supplemented with $1.5 \%$ predialyzed BSA, followed by a 30 -min chase with nonradioactive L-methionine at a final concentration of $10 \mathrm{mM}$ to allow prelabeled apoB secretion. Cells and conditioned medium were subjected to anti-apoB immunoprecipitation, followed by electrophoresis on 5\% SDS-polyacrylamide gels. After autoradiography, gel slices corresponding to apoB100 protein bands were excised from gels with the aid of autoradiogram. After dissolving in tissue solubilizer (Soluene-350; Perkin-Elmer) for $24 \mathrm{~h}$, the radioactive content of each gel slice was counted in a liquid scintillation counter (Beckman Coulter LS6500).

Fast protein liquid chromatography fractionation of lipoproteins. Aliquots $(250 \mu \mathrm{l})$ of plasma pooled from a given group of mice were applied to 2 head-to-tail linked Tricorn high-performance Superose S-6 10/300GL columns using an FPLC system (Amersham Biosciences) for the fractionation of lipoproteins, as previously described (36).

RNA isolation and real-time RT-PCR. RNA isolation from HepG2 cells or liver (20 mg) was performed using the RNeasy Mini Kit (QIAGEN). Realtime quantitative RT-PCR was used for quantifying mRNA concentrations as described in ref. 20 . The primers used were MTP forward $5^{\prime}$-TCCAGGGTGGTCTAGCTAT- $3^{\prime}$ and MTP reverse $5^{\prime}$-CCTTGTCCATCTGCATGCA$3^{\prime}$, ACOX-1 forward 5'-TCCCGATCTGCGCAAGGAGC-3' and ACOX-1 reverse $5^{\prime}$-CTGGTGAAGCAAGGTGGGCA-3', and HNF4 $\alpha$ forward 5 'CCATGGTGTTTAAGGACGTG- ${ }^{\prime}$ and HNF $4 \alpha$ reverse $5^{\prime}$-GGATCTGTTCGATCATCTGC-3'. Primers for FoxO1, PEPCK, G6Pase, SREBP-1c, FAS, ACC, $P P A R \alpha$, and $\beta$-actin mRNAs have been described $(33,39,66)$. All primers were commercially available (Integrated DNA Technologies).

Semiquantitative immunoblot assay. HepG2 cells $\left(\sim 1 \times 10^{6}\right.$ cells $)$ were lysed in $200 \mu \mathrm{l}$ M-PER (Pierce Biotechnology). To obtain protein extracts from liver tissue, $20 \mathrm{mg}$ liver tissue was homogenized in $400 \mu \mathrm{l}$ of M-PER supplemented with $4 \mu \mathrm{l}$ Halt Protease Inhibitor Cocktail (Pierce Biotechnology), followed by centrifugation at $15,000 \mathrm{~g}$ for $10 \mathrm{~min}$. Subsequent preparation of nuclear and cytosolic fractions for immunoblot analysis was performed as previously described (39) using rabbit anti-MTP antibody (1:2,000 dilution) (66) or rabbit anti-FoxO1 antibody (39). Monoclonal anti-actin antibody (1:1,000 dilution; Sigma-Aldrich) was used as control. Proteins bands were detected by autoradiography, and their relative intensities were quantified by densitometry using NIH image software as previously described (33).

Immunoprecipitation. HepG2 cells $\left(\sim 2 \times 10^{6}\right.$ cells $)$ were homogenized in $200 \mu \mathrm{l}$ of M-PER buffer supplemented with protease inhibitor cocktail (Pierce Biotechnology). After centrifugation at $15,000 \mathrm{~g}$ for $10 \mathrm{~min}$ in a microfuge, aliquots of protein lysates $(200 \mu \mathrm{g})$ were incubated with rabbit anti-apoB antibody (Abcam) for $1 \mathrm{~h}$ at room temperature, followed by immunoprecipitation, as described in ref. 66 .

Hepatic TG content. Liver tissue $(40 \mathrm{mg})$ was homogenized in $800 \mu \mathrm{l}$ of HPLC-grade acetone. After incubation with agitation at room temperature overnight, aliquots $(50 \mu \mathrm{l})$ of acetone-extract lipid suspension were used for the determination of hepatic TG content as described in ref. 39.

ChIP assay. ChIP was used to study the interaction between FoxO1 and MTP promoter DNA in cells, as previously described (36). HepG2 cells $\left(2 \times 10^{5}\right.$ cells) were transfected with pGH11 in the presence of FoxO1 vector at an MOI of $100 \mathrm{pfu} / \mathrm{cell}$ in triplicate. After 24-h incubation, cells were subjected to ChIP assay using anti-FoxO1 antibody and the ChIP assay kit (Upstate Biotechnology), as previously described (36). The immunoprecipitates were analyzed by immunoblot analysis using goat anti-FoxO1 (FKHR-C20; Santa Cruz Biotechnology Inc.), and by PCR assay to detect coimmunoprecipitated DNA using the MTP promoterspecific primers (forward 5'-GAAAAGTATCACAACTAGGT-3', reverse 5'-TGGCTCCCTCTGCCACATCCAG-3') that flank the consensus FoxO1 binding site $(-507 /+1 \mathrm{nt})$ in the mouse MTP promoter.

MTP promoter-directed luciferase reporter system. An 886-bp DNA fragment containing the mouse MTP promoter was amplified from mouse genomic DNA (BioChain Institute Inc.) by PCR using primers for forward (5'-CTAGTATCCTCAGTTGGCCT- $3^{\prime}$ ) and reverse reaction (5'-TGGCTCCCTCTGCCACATCCAG- $3^{\prime}$ ). After verifying its nucleotide sequence, the MTP promoter was cloned into the luciferase reporter pGL3-Basic vector (Promega). To generate promoter variants, DNA fragments covering different lengths 
of the MTP promoter were amplified by PCR using primers. The forward primers used were as follows: 5'-GAAAAGTATCACAACTAGGT-3' for PMTP549, 5'-CAAGATAAAAACTCCCTTGAT-3' for PMTP433, 5' -ATTAAAGGCGTGCGCCACCA-3' for PMTP255, and 5'-CTTAAAAGCGAGAGACTACA-3' for PMTP214.

Plasmid transfection and luciferase assay. HepG2 cells in 6-well microplates were transfected with $2 \mu \mathrm{g}$ of plasmid encoding the luciferase reporter gene under the control of different MTP promoter variants, using the Lipofectamine 2000 (Invitrogen). In each transfection, $2 \mu \mathrm{g}$ of plasmid pCMV$\mathrm{Lac} Z$ was included and the amount of $\beta$-gal activity was used as a control to normalize transfection efficiency. After 24-h incubation, cells were subjected to luciferase or $\beta$-gal activity assays as previously described (36).

EMSA. EMSA was used to study FoxO1 binding to DNA. FoxO1 protein was prepared from HepG2 cells pretransduced with FoxO1 vector at an MOI of $200 \mathrm{pfu} / \mathrm{cell}$. The DNA probe was derived from a 27-bp DNA covering the consensus insulin response element (IRE, -499/-473 nt) of the mouse MTP promoter (5'-TGTAGTTTGTTTTGTTTTGTTGGCATG-3'). A mutant probe contained 6 base substitutions within the IRE sequence (in lower-case letters, 5'-TGTAGTTTGcccTGcccTGTTGGCATG-3'). Oligonucleotides were labeled with biotin using the Biotin $3^{\prime}$-End DNA Labeling kit (Pierce Biotechnology), followed by self-annealing to form double-stranded biotin-labeled DNA. EMSA was performed using the
Lightshift Chemiluminescent EMSA kit (Pierce Biotechnology). For DNA supershift assay, aliquots of anti-FoxO1 antibody $(1 \mu \mathrm{g}$, goat anti-FoxO1, FKHR-C20; Santa Cruz Biotechnology Inc.) were included in the EMSA assay, as previously described (36).

Statistics. Statistical analyses of data were performed by ANOVA using StatView software (Abacus Concepts). Pair-wise comparisons were performed to study the significance between different conditions. Data are expressed as the mean \pm SEM. $P$ values less than 0.05 were considered significant.

\section{Acknowledgments}

We thank Steve Ringquist for critical reading of this manuscript. We also thank Domenico Accili for providing FoxO1 transgenics. This study was supported in part by the American Diabetes Association and NIH grant DK066301.

Received for publication June 6, 2007, and accepted in revised form April 16, 2008.

Address correspondence to: Henry Dong, Rangos Research Center, Children's Hospital of Pittsburgh, 3460 5th Avenue, Rm. 5140, Pittsburgh, Pennsylvania 15213, USA. Phone: (412) 692-6324; Fax: (412) 692-5809; E-mail: dongh@pitt.edu.
1. Ginsberg, H.N. 2003. Treatment for patients with the metabolic syndrome. Am. J. Cardiol. 91:29E-39E.

2. Moller, D.E., and Kaufman, K.D. 2005. Metabolic syndrome: a clinical and molecular perspective. Annu. Rev. Med. 56:45-62.

3. Ginsberg, H.N. 2002. New perspectives on atherogenesis: role of abnormal triglyceride-rich lipoprotein metabolism. Circulation. 106:2137-2142.

4. Avramoglu, R.K., Basciano, H., and Adeli, K. 2006. Lipid and lipoprotein dysregulation in insulin resistant states. Clin. Chim. Acta. 368:1-19.

5. Ginsberg, H.N., Zhang, Y.L., and Hernandez-Ono, A. 2005. Regulation of plasma triglycerides in insulin resistance and diabetes. Arch. Med. Res. 36:232-240.

6. Lewis, G.F., Uffelman, K.D., Szeto, L.W., Weller, B., and Steiner, G. 1995. Interaction between free fatty acids and insulin in the acute control of very low density lipoprotein production in humans. J. Clin. Invest. 95:158-166.

7. den Boer, M.A., Voshol, P.J., Kuipers, F., Romijn, J.A., and Havekes, L.M. 2006. Hepatic glucose production is more sensitive to insulin-mediated inhibition than hepatic VLDL-triglyceride production. Am. J. Physiol. Endocrinol. Metab. 291:E1360-E1364.

8. Wiegman, C.H., et al. 2003. Hepatic VLDL production in ob/ob mice is not stimulated by massive de novo lipogenesis but is less sensitive to the suppressive effects of insulin. Diabetes. 52:1081-1089.

9. Hussain, M.M., Shi, J., and Dreizen, P. 2003. Microsomal triglyceride transfer protein and its role in apoB-lipoprotein assembly. J. Lipid Res. 44:22-32.

10. Manchekar, M., et al. 2004. Apolipoprotein B-containing lipoprotein particle assembly: lipid capacity of the nascent lipoprotein particle. J. Biol. Chem. 279:39757-39766.

11. Swift, L.L., Jovanovska, A., Kakkad, B., and Ong, D.E. 2005. Microsomal triglyceride transfer protein expression in mouse intestine. Histochem. Cell Biol. 123:475-482.

12. Berriot-Varoqueaux, N., Aggerbeck, L.P., SamsonBouma, M., and Wetterau, J.R. 2000. The role of the microsomal triglygeride transfer protein in abetalipoproteinemia. Annu. Rev. Nutr. 20:663-697.

13. Liao, W., Hui, T.Y., Young, S.G., and Davis, R.A. 2003. Blocking microsomal triglyceride transfer protein interferes with apoB secretion without causing retention or stress in the ER. J. Lipid Res.
44:978-985.

14. Bjorkegren, J., Beigneux, A., Bergo, M.O., Maher, J.J., and Young, S.G. 2002. Blocking the secretion of hepatic very low density lipoproteins renders the liver more susceptible to toxin-induced injury. J. Biol. Chem. 277:5476-5483.

15. Leung, G.K., et al. 2000. A deficiency of microsomal triglyceride transfer protein reduces apolipoprotein B secretion. J. Biol. Chem. 275:7515-7520.

16. Raabe, M., et al. 1999. Analysis of the role of microsomal triglyceride transfer protein in the liver of tissue-specific knockout mice. J. Clin. Invest. 103:1287-1298.

17. Tietge, U.J., et al. 1999. Hepatic overexpression of microsomal triglyceride transfer protein (MTP) results in increased in vivo secretion of VLDL triglycerides and apolipoprotein B. J. Lipid Res. 40:2134-2139.

18. Cuchel, M., et al. 2007. Inhibition of microsomal triglyceride transfer protein in familial hypercholesterolemia. N. Engl. J. Med. 356:148-156.

19. Day, C.P. 2006. Genes or environment to determine alcoholic liver disease and non-alcoholic fatty liver disease. Liver Int. 26:1021-1028.

20. Phillips, C., Owens, D., Collins, P., and Tomkin, G.H. 2002. Microsomal triglyceride transfer protein: does insulin resistance play a role in the regulation of chylomicron assembly? Atherosclerosis. 160:355-360

21. Avramoglu, R.K., and Adeli, K. 2004. Hepatic regulation of apolipoprotein B. Rev. Endocr. Metab. Disord. 5:293-301.

22. Au, W.S., Kung, H.F., and Lin, M.C. 2003. Regulation of microsomal triglyceride transfer protein gene by insulin in HepG2 cells: roles of MAPKerk and MAPKp38. Diabetes. 52:1073-1080.

23. Hagan, D.L., Kienzle, B., Jamil, H., and Hariharan, N. 1994. Transcriptional regulation of human and hamster microsomal triglyceride transfer protein genes. Cell type-specific expression and response to metabolic regulators. J. Biol. Chem. 269:28737-28744.

24. Lin, M.C., Gordon, D., and Wetterau, J.R. 1995. Microsomal triglyceride transfer protein (MTP) regulation in HepG2 cells: insulin negatively regulates MTP gene expression. J. Lipid Res. 36:1073-1081.

25. Allister, E.M., Borradaile, N.M., Edwards, J.Y., and Huff, M.W. 2005. Inhibition of microsomal triglyceride transfer protein expression and apolipoprotein B100 secretion by the citrus flavonoid naringenin and by insulin involves activation of the mitogen-activated protein kinase pathway in hepatocytes. Diabetes. 54:1676-1683.

26. Siri, P., et al. 2001. Post-transcriptional stimulation of the assembly and secretion of triglyceride-rich apolipoprotein B lipoproteins in a mouse with selective deficiency of brown adipose tissue, obesity, and insulin resistance. J. Biol. Chem. 276:46064-46072.

27. Ota, T., Gayet, C., and Ginsberg, H.N. 2008. Inhibition of apolipoprotein B100 secretion by lipidinduced hepatic endoplasmic reticulum stress in rodents. J. Clin. Invest. 118:316-332.

28. Arden, K.C. 2004. FoxO: linking new signaling pathways. Mol. Cell. 14:416-418.

29. Barthel, A., Schmoll, D., and Unterman, T.G. 2005. FoxO proteins in insulin action and metabolism. Trends Endocrinol. Metab. 16:183-189.

30. Matsumoto, M., Pocai, A., Rossetti, L., Depinho, R.A., and Accili, D. 2007. Impaired regulation of hepatic glucose production in mice lacking the forkhead transcription factor foxo 1 in liver. Cell Metab. 6:208-216.

31. Nakae, J., Kitamura, T., Ogawa, Y., Kasuga, M., and Accili, D. 2001. Insulin regulation of gene expression through the forkhead transcription factor Foxo1 (Fkhr) requires kinases distinct from Akt. Biochemistry. 40:11768-11776.

32. Nakae, J., Barr, V., and Accili, D. 2000. Differential regulation of gene expression by insulin and IGF-1 receptors correlates with phosphorylation of a single amino acid residue in the forkhead transcription factor FKHR. EMBOJ. 19:989-996.

33. Altomonte, J., et al. 2003. Inhibition of Foxo1 function is associated with improved fasting glycemia in diabetic mice. Am. J. Physiol. Endocrinol. Metab. 285:E718-E728.

34. O’Brien, R.M., Streeper, R.S., Ayala, J.E., Stadelmaier, B.T., and Hornbuckle, L.A. 2001. Insulinregulated gene expression. Biochem. Soc. Trans. 29:552-558.

35. Nakae, J., et al. 2002. Regulation of insulin action and pancreatic $\beta$-cell function by mutated alleles of the gene encoding forkhead transcription factor Foxo1. Nat. Genet. 32:245-253.

36. Altomonte, J., et al. 2004. Foxo1 mediates insulin 
action on ApoC-III and triglyceride metabolism. J. Clin. Invest. 114:1493-1503.

37. Ameen, C., et al. 2005. Activation of peroxisome proliferator-activated receptor alpha increases the expression and activity of microsomal triglyceride transfer protein in the liver. J. Biol. Chem. 280:1224-1229.

38. Knapp, J.E., and Liu, D. 2004. Hydrodynamic delivery of DNA. Methods Mol Biol 245:245-250.

39. Qu, S., et al. 2006. Aberrant Forkhead box O1 function is associated with impaired hepatic metabolism. Endocrinology. 147:5641-5652.

40. Accili, D., and Arden, K.C. 2004. FoxOs at the crossroads of cellular metabolism, differentiation, and transformation. Cell. 117:421-426.

41. Nakae, J., Park, B.-C., and Accili, D. 1999. Insulin stimulates phosphorylation of the forkhead transcription factor FKHR on serine 253 through a wortmannin-sensitive pathway. J. Biol. Chem. 274:15982-15985.

42. Chirieac, D.V., et al. 2000. Glucose-stimulated insulin secretion suppresses hepatic triglyceride-rich lipoprotein and apoB production. Am. J. Physiol. Endocrinol. Metab. 279:E1003-E1011.

43. Lewis, G.F. 1997. Fatty acid regulation of very low density lipoprotein production. Curr. Opin. Lipidol. 8:146-153.

44. Steiner, G., and Lewis, G.F. 1996. Hyperinsulinemia and triglyceride-rich lipoproteins. Diabetes. 45(Suppl. 3):S24-S26.

45. Durrington, P.N., Newton, R.S., Weinstein, D.B. and Steinberg, D. 1982. Effects of insulin and glucose on very low density lipoprotein triglyceride secretion by cultured rat hepatocytes. J. Clin. Invest. 70:63-73.

46. Sparks, C.E., et al. 1986. Insulin effects on apolipoprotein B lipoprotein synthesis and secretion by primary cultures of rat hepatocytes. Metabolism. 35:1128-1136.

47. Patsch, W., Franz, S., and Schonfeld, G. 1983. Role of insulin in lipoprotein secretion by cultured rat hepatocytes. J. Clin. Invest. 71:1161-1174.

48. Sparks, J.D., and Sparks, C.E. 1990. Insulin modulation of hepatic synthesis and secretion of apolipoprotein B by rat hepatocytes. J. Biol. Chem. 265:8854-8862.

49. Pullinger, C.R., et al. 1989. The apolipoprotein B gene is constitutively expressed in HepG2 cells: regulation of secretion by oleic acid, albumin, and insulin, and measurement of the mRNA half-life. J. Lipid Res. 30:1065-1077.

50. Dashti, N., Williams, D.L., and Alaupovic, P. 1989. Effects of oleate and insulin on the production rates and cellular mRNA concentrations of apolipoproteins in HepG2 cells. J. Lipid Res. 30:1365-1373.

51. Sparks, J.D., Sparks, C.E., and Miller, L.L. 1989. Insulin effects on apolipoprotein B production by normal, diabetic and treated-diabetic rat liver and cultured rat hepatocytes. Biochem. J. 261:83-88.
52. Stein, D.T., et al. 1996. Essentiality of circulating fatty acids for glucose-stimulated insulin secretion in the fasted rat. J. Clin. Invest. 97:2728-2735.

53. Holm, C., Osterlund, T., Laurell, H., and Contreras, J.A. 2000. Molecular mechanisms regulating hormone-sensitive lipase and lipolysis. Annu. Rev. Nutr. 20:365-393.

54. Malmstrom, R., et al. 1998. Effects of insulin and acipimox on VLDL1 and VLDL2 apolipoprotein B production in normal subjects. Diabetes. 47:779-787.

55. Malmstrom, R., et al. 1997. Metabolic basis of hypotriglyceridemic effects of insulin in normal men. Arterioscler. Thromb. Vasc. Biol. 17:1454-1464.

56. Wolfrum, C., Asilmaz, E., Luca, E., Friedman, J.M., and Stoffel, M. 2004. Foxa2 regulates lipid metabolism and ketogenesis in the liver during fasting and in diabetes. Nature. 432:1027-1032.

57. Wolfrum, C., and Stoffel, M. 2006. Coactivation of Foxa2 through Pgc-1beta promotes liver fatty acid oxidation and triglyceride/VLDL secretion. Cell. Metab. 3:99-110.

58. Lin, J., et al. 2005. Hyperlipidemic effects of dietary saturated fats mediated through PGC-1beta coactivation of SREBP. Cell. 120:261-273.

59. Hall, R.K., et al. 2000. Regulation of phosphoenlpyruvate carboxykinase and insulin-like growth factor-binding protein-1 gene expression by insulin. J. Biol. Chem. 275:30169-30175.

60. Wang, J.C., Stromstedt, P.E., O'Brien, R.M., and Granner, D.K. 1996. Hepatic nuclear factor 3 is an accessory factor required for the stimulation of phosphoenolpyruvate carboxykinase gene transcription by glucocorticoids. Mol. Endocrinol. 10:794-800.

61. Powell, D.R., et al. 1995. Multiple proteins bind the insulin response element in the human IGFBP-1 promoter. Prog. Growth Factor Res. 6:93-101.

62. O'Brien, R.M., et al. 1995. Hepatic nuclear factor 3- and hormone-regulated expression of the phosphoenolpyruvate carboxykinase and insulin-like growth factor-binding protein 1 genes. Mol. Cell. Biol. 15:1747-1758.

63. Roux, J., Pictet, R., and Grange, T. 1995. Hepatocyte nuclear factor 3 determines the amplitude of the glucocorticoid response of the rat tyrosine aminotransferase gene. DNA Cell Biol. 14:385-396.

64. Zhang, L., Rubins, N.E., Ahima, R.S., Greenbaum, L.E., and Kaestner, K.H. 2005. Foxa2 integrates the transcriptional response of the hepatocyte to fasting. Cell Metab. 2:141-148.

65. Friedman, J.R., and Kaestner, K.H. 2006. The Foxa family of transcription factors in development and metabolism. Cell. Mol. Life Sci. 63:2317-2328.

66. Qu, S., et al. 2007. PPAR \{alpha\} mediates the hypolipidemic action of fibrates by antagonizing FoxO1. Am. J. Physiol. Endocrinol. Metab. 292:E421-E434.

67. Bartels, E.D., Lauritsen, M., and Nielsen, L.B. 2002 Hepatic expression of microsomal triglyceride trans- fer protein and in vivo secretion of triglyceride-rich lipoproteins are increased in obese diabetic mice. Diabetes. 51:1233-1239.

68. Zhang, Y.L., et al. 2004. Regulation of hepatic apolipoprotein B-lipoprotein assembly and secretion by the availability of fatty acids. I. Differential response to the delivery of fatty acids via albumin or remnant-like emulsion particles. J. Biol. Chem. 279:19362-19374.

69. Matsumoto, M., Han, S., Kitamura, T., and Accili, D. 2006. Dual role of transcription factor FoxO1 in controlling hepatic insulin sensitivity and lipid metabolism. J. Clin. Invest. 116:2464-2472.

70. Valenti, L., et al. 2008. Increased expression and activity of the transcription factor Foxo1 in nonalcoholic steatohepatitis. Diabetes. Online publication ahead of print.

71. Zhang, W., et al. 2006. FoxO1 regulates multiple metabolic pathways in the liver: effects on gluconeogenic, glycolytic, and lipogenic gene expression. J. Biol. Chem. 281:10105-10117.

72. Biddinger, S.B., et al. 2008. Hepatic insulin resistance is sufficient to produce dyslipidemia and susceptibility to atherosclerosis. Cell Metab. 7:125-134.

73. Stiles, B., et al. 2004. Liver-specific deletion of negative regulator Pten results in fatty liver and insulin hypersensitivity [erratum 2004, 101:5180]. Proc. Natl. Acad. Sci. U. S. A. 101:2082-2087.

74. Horie, Y., et al. 2004. Hepatocyte-specific Pten deficiency results in steatohepatitis and hepatocellular carcinomas. J. Clin. Invest. 113:1774-1783.

75. Rao, P., et al. 2005. Gene transfer of constitutively active Akt markedly improves human islet transplant outcomes in diabetic severe combined immunodeficient mice. Diabetes. 54:1664-1675.

76. Bernal-Mizrachi, E., et al. 2001. Islet beta cell expression of constitutively active Akt1/PKB alpha induces striking hypertrophy, hyperplasia, and hyperinsulinemia. J. Clin. Invest. 108:1631-1638.

77. Shoelson, S.E., Chatterjee, S., Chaudhuri, M., and White, M.F. 1992. YMXM motifs of IRS-1 define substrate specificity of the insulin receptor kinase. Proc. Natl. Acad. Sci. U. S. A. 89:2027-2031.

78. Garcia, P., et al. 1993. Phosphorylation of synthetic peptides containing Tyr-Met-X-Met motifs by nonreceptor tyrosine kinases in vitro. J. Biol. Chem. 268:25146-25151.

79. Rordorf-Nikolic, T., Van Horn, D.J., Chen, D., White, M.F., and Backer, J.M. 1995. Regulation of phosphatidylinositol 3'-kinase by tyrosyl phosphoproteins. Full activation requires occupancy of both $\mathrm{SH} 2$ domains in the $85-\mathrm{kD}$ a regulatory subunit. J. Biol. Chem. 270:3662-3666.

80. Borradaile, N.M., de Dreu, L.E., and Huff, M.W. 2003. Inhibition of net HepG2 cell apolipoprotein $\mathrm{B}$ secretion by the citrus flavonoid naringenin involves activation of phosphatidylinositol 3kinase, independent of insulin receptor substrate-1 phosphorylation. Diabetes. 52:2554-2561. 